\title{
Numerical simulations of mid-ocean ridge hydrothermal circulation including the phase separation of seawater
}

\author{
Yoshifumi Kawada, Shigeo Yoshida, and Sei-ichiro Watanabe \\ Department of Earth and Planetary Sciences, Nagoya University, Furo-cho, Chikusa-ku, Nagoya 464-8602, Japan
}

(Received September 8, 2003; Revised January 16, 2004; Accepted January 27, 2004)

\begin{abstract}
Phase separation of seawater is an important process controlling the dynamics and chemistry of hydrothermal circulation. We numerically investigate hydrothermal circulation in porous media, including phase separation of seawater. Seawater enters the crust at the seafloor, is heated at depth, and returns to the seafloor as hydrothermal fluids. The seafloor and the bottom of the calculation region are set at depths of $2500 \mathrm{~m}$ and $4000 \mathrm{~m}$ from the sea surface, respectively. The temperature at the base of the calculation region is set at $600^{\circ} \mathrm{C}$. Under these pressure and temperature ranges, supercritical phase separation is inevitable. Here we focus on steady-state conditions, as a first step to investigate the complex process of convection with phase separation. Under these conditions, we demonstrate that phase separation leads to a two-layer structure. Seawater circulates vigorously in the upper layer, and this overlies a stagnant lower layer formed by sinking of dense brine. We find that the key quantity which governs this structure is the ratio of the relative velocity between the two phases to the mean flow velocity in the transition zone between the two layers. As the relative velocity increases, the brine layer becomes thick, and the transition zone becomes thin. Under steady state conditions, the mean salinity at the seafloor should be the same as that of seawater because the total mass of salt should be conserved. Fluids which vent near the ridge axis are more saline than seawater, whereas fluids which vent more than about $100 \mathrm{~m}$ away from the axis are less saline than seawater.
\end{abstract}

Key words: Hydrothermal circulation, phase separation, porous media.

\section{Introduction}

Around mid-ocean ridges, seawater sinks through cracks of oceanic crusts and flows out near the crests, being driven by the heat of underlying cooling magma chambers. The outflowing hot water forms spectacular black smoker vents in some places. The flow is called mid-ocean ridge hydrothermal circulation (e.g. Kelley et al., 2002). The circulation plays a major role in heat transport at ridge areas. Moreover, it nourishes deep-sea biotic communities, and causes hydrothermal alterations, which are commonly found in ophiolites. All of these phenomena are influenced by the dynamics of the circulation. For example, ecosystems are controlled by the temperature and composition of the fluids, which result from heat transport and chemical reactions, which are in turn determined by the circulation structure.

The numerical simulation is a powerful tool for investigating such complex phenomena. Many simulations have been carried out for the systems which are basically thermal convection in porous media. Early simulations used the properties of pure water (e.g. Fehn et al., 1983; Travis et al., 1991) for hydrothermal fluids. Effects of more realistic material properties of hydrothermal fluids have been investigated recently. Wilcock (1998) used temperature- and pressure-dependent properties of seawater to investigate the temperature of the discharging fluid. These properties of

Copy right (c) The Society of Geomagnetism and Earth, Planetary and Space Sciences (SGEPSS); The Seismological Society of Japan; The Volcanological Society of Japan; The Geodetic Society of Japan; The Japanese Society for Planetary Sciences; TERRAPUB. seawater, especially its critical properties, give rise to the focusing of upwelling. He showed that the temperature of the discharging fluid is about half of the bottom temperature. The calculated venting temperature is reasonably close to observed temperatures of black smokers, $320-380^{\circ} \mathrm{C}$. He however noted that the temperature is sensitive to the permeability structure. Jupp and Schultz (2000) used the equation of state of pure water to investigate the effect of anomalous physical properties of water near its critical point, $374^{\circ} \mathrm{C}$, on the temperature of black smokers. They used numerical simulations to demonstrate that thermal plumes tend to form at temperatures of about $400^{\circ} \mathrm{C}$, which is close to the critical temperature, where physical properties show anomalous behavior.

Phase separation of seawater is another factor that has profound influence on the physical properties of hydrothermal fluids, and it has been considered to be a major controlling factor of the circulation structure (Bischoff and Rosenbauer, 1989). It is particularly important around ridge axes, where the underlying magma chamber heats seawater above the critical point. The phase separation generates fluids with $\mathrm{Cl}^{-}$ concentrations both higher and lower than that of seawater, and leads to the change of $\mathrm{Cl}^{-}$concentrations of hydrothermal vent fluids. The evidence of phase separation comes from both the chemical compositions of mid-ocean ridge fluids and fluid inclusions in ophiolites.

The diversity of $\mathrm{Cl}^{-}$concentrations of hydrothermal vent water (e.g. Von Damm, 1995) is considered to result from phase separation. The $\mathrm{NaCl}$ concentrations range from 0.2 


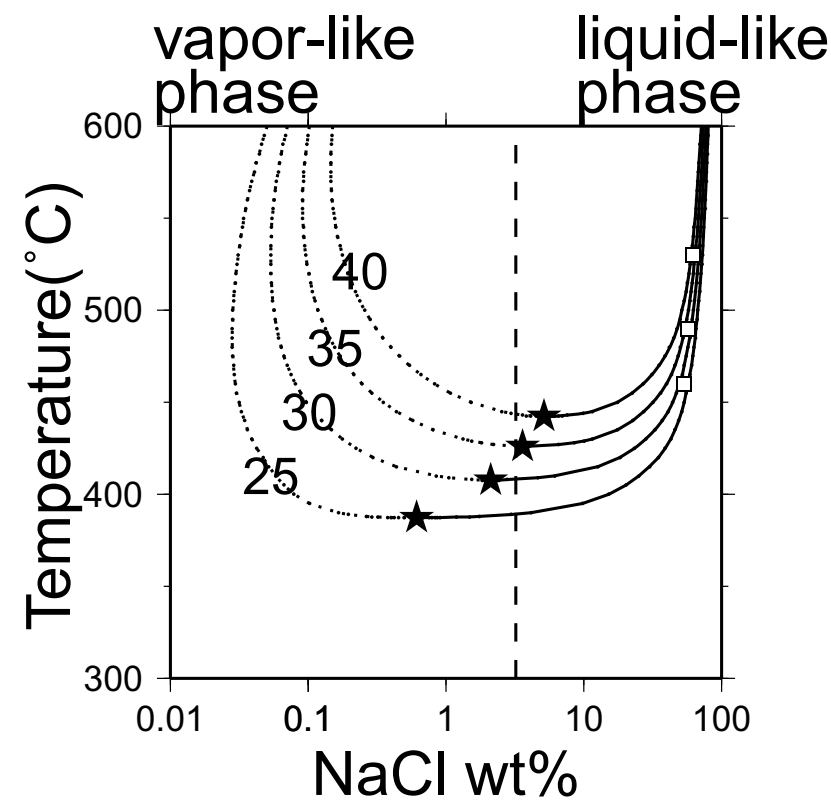

Fig. 1. Two-phase (liquid-vapor) coexisting curve of $\mathrm{NaCl}-\mathrm{H}_{2} \mathrm{O}$ twocomponent fluid, based on the theory of Anderko and Pitzer (1993). The vertical axis is the temperature in Celsius degree, and the horizontal axis is the logarithm of the $\mathrm{NaCl}$ concentration in wt $\%$. The solid curves show the $\mathrm{NaCl}$ concentrations of the liquid-like phase, and the dotted curves show those of the vapor-like phase at various pressures. The numbers on the curves are the pressures in MPa. The stars represent the critical points for those pressures. The fluid below these critical temperatures is one-phase. The vertical dashed line shows the $\mathrm{NaCl}$ concentration of seawater. The open squares show the halite-liquid-vapor triple point for pressures of 25, 30, and $35 \mathrm{MPa}$ (Palliser and McKibbin, 1998a). The triple point does not exist for $40 \mathrm{MPa}$. Halite-saturation curves are not shown since they are not relevant in this study.

to $6 \mathrm{wt} \%$, whereas seawater has a $\mathrm{NaCl}$ concentration of 3.2 $\mathrm{wt} \%$. There are two possible mechanisms that can give rise to the variation: phase separation and water-rock reactions. Phase separation is considered to be dominant because no water-rock reactions are known to be effective in changing $\mathrm{Cl}^{-}$concentrations (Bischoff and Rosenbauer, 1989). Moreover, the linear relationship between observed $\mathrm{Cl}$ and $\mathrm{Br}$ concentrations supports the phase separation mechanism, because $\mathrm{Br}$ and $\mathrm{Cl}$ behave in a similar way upon phase separation (Berndt and Seyfried, 1990), and that $\mathrm{Br}$ would be excluded from the most probable Cl-bearing phase $\mathrm{Fe}_{2}(\mathrm{OH})_{3} \mathrm{Cl}$ that can give rise to observed $\mathrm{Cl}$ variations.

Fluid inclusions in ophiolites also support the presence of phase separation in hydrothermal systems. According to the compilation of Kelley et al. (1992) (their figure 1), fluid inclusions are grouped into two distinct clusters, one having salinities below $20 \mathrm{wt} \% \mathrm{NaCl}$ equivalent (mostly below 10 $\mathrm{wt} \%$ ), and the other having salinities above $30 \mathrm{wt} \% \mathrm{NaCl}$ equivalent. The high-salinity group has trapping temperatures above $400^{\circ} \mathrm{C}$. This is consistent with the phase diagram of the $\mathrm{NaCl}-\mathrm{H}_{2} \mathrm{O}$ system (Fig. 1), because the phase separation of seawater, which is essential in producing brine, occurs at temperatures above $400^{\circ} \mathrm{C}$ when the pressure is above 250 bar. Fluid inclusions with lower trapping temperatures have low salinities, and most low-salinity inclusions have trapping temperatures below $400^{\circ} \mathrm{C}$. Assuming temperature increases with depth, the above result indicates the existence of two- layer hydrothermal circulation with a low-salinity layer overlying a brine layer, as suggested by Bischoff and Rosenbauer (1989).

The importance of phase separation has led some researchers to extend their numerical simulations to include its effect, though its treatment is not complete so far because of various numerical difficulties. Two types of models have been used to incorporate the effect of phase separation: two-dimensional convection in porous media, and one-dimensional pipe flow.

The two-dimensional convection simulation is a powerful tool for illustrating underground flow, but phase separation effect is not fully included so far. Schoofs and Hansen (2000) calculated the depletion of the bottom brine layer of twolayer convection as envisaged by Bischoff and Rosenbauer (1989). They modeled the convection just after the fluid becomes one-phase due to the decline of the heat source. They prescribed $\mathrm{NaCl}$ concentration contrasts between the upper and lower layers, and investigated how the lower layer breaks down. They found two mechanisms for the breakdown of the bottom layer: convective breakdown and interface migration. Lowell and Xu (2000) examined the time evolution of hydrothermal circulation when a dike intrudes. They used a phase diagram which includes two-phase separation. One of their results is concerned with $\mathrm{NaCl}$ concentrations of hydrothermal fluids at the seafloor. The vent fluid is dilute at first because seawater vaporizes. After about a month, the two-phase region disappears and the $\mathrm{NaCl}$ concentration of the vent fluid returns to that of seawater. However, transport of salt is not explicitly treated in their calculation.

One-dimensional pipe models have been used in some studies because phase separation is difficult to treat in the calculation of convection. The idea is to simplify the dynamics and focus on the effect of phase separation. Lowell and Germanovich (1997) treated time-dependent behavior of a brine layer by one-dimensional modeling. They described a rough picture of the evolution, by estimating the timescales of generation and depletion of the brine layer. Bai et al. (2003) were the first to calculate phase separation process in a heat-pipe model. They numerically calculated how phase separation proceeds to develop a brine layer. Their results are closely related to our one-dimensional analysis in Section 5, where we give more detailed explanation of salinity distribution than theirs.

Our work is of the first type, namely, two-dimensional numerical simulations of thermal convection in porous media, and we include the effect of phase separation in a consistent manner, including the transport of salt and the conservation of its total mass. The purposes of this work are to reveal the flow structure of hydrothermal circulation undergoing phase separation, to clarify the physical mechanism of the structure formation, and to identify key quantities which are essential to the structure. For those purposes, we carry out simulations of convection driven by the heat conducted from the underlying magma chamber, including supercritical phase separation of $\mathrm{NaCl}-\mathrm{H}_{2} \mathrm{O}$ two-component system. We carry out detailed analyses of the sensitivity to relevant parameters to identify key parameters.

We calculate steady-state solutions as a first step to investigate this complex process of convection with phase separa- 
tion. The meaning of the word "steady" is twofold. One is that transient phenomena, for example, caused by intrusion of magma, are not treated in this study. The other is that flow is not turbulent because we use relatively low Rayleigh numbers to ensure numerical reliability of the results as explained in Section 3. Therefore some caution must be exercised when we compare our results with real systems. For the first meaning of steadiness, we envisage fast spreading ridges, where magma chambers exist continuously along the ridge axis and continuously in time (e.g. Sinton and Detrick, 1992). Some vents at these ridges emit high chlorinity fluids stably for over a decade (e.g. Von Damm, 1995), and this shows that stable conditions do exist at some sites. For the second meaning of steadiness, if the Rayleigh numbers at the ridge axis area are high, the convection should be highly time-dependent (e.g. Schoofs et al., 1999; Schoofs and Spera, 2003). For high Rayleigh number conditions, our results may be considered as a metaphor to real systems, if our low Rayleigh number results can be interpreted as a statistical mean, as in eddy diffusivity formulation of the theory of turbulence. Our detailed analyses allow us to interpret observations at least qualitatively. The temperature and $\mathrm{NaCl}$ concentration of hydrothermal fluids at the seafloor are of interest in relation to observations, and discussions will be given on what we can infer about the circulation structure and phase separation from their observations.

This paper is structured as follows. In Section 2, we derive governing equations for two-phase two-component flow in porous media and the appropriate boundary conditions. Section 3 describes the framework of the numerical calculation and the adopted values of the physical parameters. The results of the two-dimensional numerical calculations are shown in Section 4. Section 5 presents a one-dimensional analysis, which provides a better understanding of the structure of the two-phase region. We discuss the relationship of our results with real systems in Section 6, and concluding remarks follow in Section 7.

\section{Equations and Boundary Conditions \\ 2.1 Governing equations}

We derive the equations for two-phase two-component flow in porous media. The two phases are the vapor and liquid phases of the $\mathrm{NaCl}-\mathrm{H}_{2} \mathrm{O}$ two-component system. The basic equations consist of four conservation equations (mass, momentum, energy, $\mathrm{NaCl}$ concentration), a constitutive equation (the relative velocity between the two phases), the equation of state (density of the liquid and vapor phases) and the phase diagram (liquid-vapor co-existing curve) of the $\mathrm{NaCl}-\mathrm{H}_{2} \mathrm{O}$ system.

We use simplified conservation equations, which are derived in the following way. First, we write conservation equations of a conservative quantity for the relevant phases (the liquid, vapor, and rock matrix for the energy equation, the liquid and vapor for other equations). Second, we add the equations for these phases to obtain an equation for the mixture. The mixture equation is convenient when we treat both one-phase and two-phase regions, because we can use the same equation for both regions. The basic idea of this approach is the same as that of the diffusion model approach for two-phase fluid often used in engineering problems (e.g.
Ishii, 1975) (see Section 2.1.2). Third, the Boussinesq approximation is applied to the resulting equation for simplicity; we use the equation of state described in Section 2.1.6 for the density variation of the buoyancy term, and treat the densities in the other terms as a constant. We apply the Boussinesq approximation to the equations because we intend to single out the effect of the relative separation between the liquid and vapor phases. The Boussinesq approximation is sufficient for obtaining a qualitative picture of thermal convection, when the role of sound waves is not important. Fourth, other approximations are applied. The temperature and pressure dependence of the other material properties (viscosity, thermal conductivity, heat capacity, and diffusion coefficient of $\mathrm{NaCl}$ ), and the latent heat of condensation are neglected. We treat the porosity and permeability as constants. We use a simple hydrostatic relationship for the pressure in the calculations of the equation of state and the phase diagram of the two-phase fluid. The justification of each approximation is discussed in the following subsections.

2.1.1 Mass conservation The mass conservation equation for the liquid phase is written as

$$
\frac{\partial}{\partial t}\left(\phi \psi_{1} \rho_{1}\right)+\nabla \cdot\left(\phi \psi_{1} \rho_{1} v_{1}\right)=\Gamma
$$

and that for the vapor phase is written as

$$
\frac{\partial}{\partial t}\left[\phi\left(1-\psi_{1}\right) \rho_{\mathrm{v}}\right]+\nabla \cdot\left[\phi\left(1-\psi_{1}\right) \rho_{\mathrm{v}} \boldsymbol{v}_{\mathrm{v}}\right]=-\Gamma,
$$

where $t$ is time, $\phi$ is the porosity, $\psi_{1}$ is the volume fraction of the liquid phase, $v_{1}$ is the velocity of the liquid phase, $v_{\mathrm{v}}$ is the velocity of the vapor phase, $\rho_{1}$ is the density of the liquid phase, $\rho_{\mathrm{v}}$ is the density of the vapor phase, and $\Gamma$ denotes the mass production term representing the phase change from vapor to liquid. Note that the velocities and the Darcy fluxes are related as $\boldsymbol{Q}_{1}=\phi \psi_{1} \boldsymbol{v}_{\mathrm{l}}, \boldsymbol{Q}_{\mathrm{v}}=\phi\left(1-\psi_{1}\right) \boldsymbol{v}_{\mathrm{v}}$, where $\boldsymbol{Q}_{\mathrm{l}}$ is the Darcy flux of the liquid phase, and $\boldsymbol{Q}_{\mathrm{v}}$ is that of the vapor phase (e.g. Bear, 1988).

The mixture equation is obtained by adding equations (1) and (2) as

$$
\frac{\partial}{\partial t}\left(\phi \rho_{\mathrm{f}}\right)+\boldsymbol{\nabla} \cdot\left(\phi \rho_{\mathrm{f}} \boldsymbol{v}\right)=0
$$

where $v$ is the local center-of-mass velocity defined as

$$
\boldsymbol{v}=\omega_{1} \boldsymbol{v}_{\mathrm{l}}+\left(1-\omega_{1}\right) \boldsymbol{v}_{\mathrm{v}},
$$

and the mean density of the fluid $\rho_{\mathrm{f}}$ is defined as

$$
\rho_{\mathrm{f}}=\psi_{\mathrm{l}} \rho_{\mathrm{l}}+\left(1-\psi_{1}\right) \rho_{\mathrm{v}}
$$

Here $\omega_{1}$ is the mass fraction of the liquid phase. Note that the liquid mass fraction $\omega_{1}$ and the liquid volume fraction $\psi_{1}$ are related as

$$
\omega_{1}=\frac{\rho_{1}}{\rho_{\mathrm{f}}} \psi_{1}
$$

With the Boussinesq approximation and constant porosity, the mass conservation equation (3) is simplified to

$$
\boldsymbol{\nabla} \cdot \boldsymbol{v}=0
$$


2.1.2 Momentum conservation To derive momentum equations, we use the idea of the diffusion model approach often used in engineering problems (e.g. Ishii, 1975), instead of using the relative permeability model often used in ground water or hydrothermal problems. We start with the momentum conservation equation for each phase. The momentum conservation equation for the liquid phase is written as

$$
\begin{aligned}
0= & -\phi \psi_{1} \nabla p+\phi \psi_{1} \rho_{1} g \\
& -\phi \psi_{1} \frac{\mu_{1}}{K_{1}} \phi v_{1}-\phi \psi_{1}\left(1-\psi_{1}\right) L_{0} \phi\left(v_{1}-v_{v}\right),
\end{aligned}
$$

and the equation for the vapor phase is written as

$$
\begin{aligned}
0= & -\phi\left(1-\psi_{1}\right) \nabla p+\phi\left(1-\psi_{1}\right) \rho_{\mathrm{v}} \boldsymbol{g} \\
& -\phi\left(1-\psi_{1}\right) \frac{\mu_{\mathrm{v}}}{K_{\mathrm{v}}} \phi \boldsymbol{v}_{\mathrm{v}}-\phi \psi_{1}\left(1-\psi_{1}\right) L_{0} \phi\left(\boldsymbol{v}_{\mathrm{v}}-v_{1}\right),
\end{aligned}
$$

where $p$ is the pressure, $g$ is the gravitational acceleration (whose direction is downward), $\mu_{1}$ is the viscosity of the liquid phase, $\mu_{\mathrm{v}}$ is the viscosity of the vapor phase, $K_{1}$ is the permeability of the liquid phase, $K_{\mathrm{v}}$ is the permeability of the vapor phase, and $L_{0}$ denotes the friction coefficient between the two phases. In these equations, we assume that the pressures of the liquid and the vapor are the same at the same macroscopic point. The first term of the liquid phase equation (8) is the force which results from the pressure gradient of the liquid phase, the second term is the gravitational body force on the liquid phase, the third term is the drag between the liquid and the rock matrix, and the fourth term is the resistance between the liquid and vapor. Each term of the vapor phase equation (9) has a meaning similar to the corresponding term in the liquid phase equation (8). Note that the fourth terms of these equations should have the same magnitude with the opposite sign, because of the law of action and reaction.

The momentum conservation equations (8), (9) have a form different from the commonly accepted Darcy's law which makes use of the notion of the relative permeability (e.g. Bear, 1988; Lowell, 1991; Nield and Bejan, 1999),

$$
\begin{aligned}
& \boldsymbol{Q}_{\mathrm{l}}=-\frac{k_{\mathrm{r}, 1} K_{0}}{\mu_{\mathrm{l}}}\left(\nabla p-\rho_{\mathrm{l}} \boldsymbol{g}\right), \\
& \boldsymbol{Q}_{\mathrm{v}}=-\frac{k_{\mathrm{r}, \mathrm{v}} K_{0}}{\mu_{\mathrm{v}}}\left(\nabla p-\rho_{\mathrm{v}} \boldsymbol{g}\right),
\end{aligned}
$$

where $K_{0}$ is the permeability, and $k_{\mathrm{r}, \mathrm{l}}$ and $k_{\mathrm{r}, \mathrm{v}}$ are the relative permeabilities of the liquid and the vapor, respectively. To compare these forms and ours, we rearrange equations (8) and (9) into the form similar to equations (10) and (11) by solving them in terms of $\boldsymbol{Q}_{1}$ and $\boldsymbol{Q}_{\mathrm{v}}$ as

$$
\left(\begin{array}{c}
\boldsymbol{Q}_{1} \\
\boldsymbol{Q}_{\mathrm{v}}
\end{array}\right)=-\frac{\tilde{K}}{\mu_{\mathrm{eff}}}\left(\begin{array}{c}
\boldsymbol{\nabla} p-\rho_{\mathrm{l}} \boldsymbol{g} \\
\nabla p-\rho_{\mathrm{v}} \boldsymbol{g}
\end{array}\right),
$$

where $\tilde{K}$ is the permeability matrix which is defined as

$$
\begin{gathered}
\frac{\tilde{K}}{\mu_{\text {eff }}}=\frac{1}{\left\{\frac{\mu_{1}}{K_{1}}+\left(1-\psi_{1}\right) L_{0}\right\}\left\{\frac{\mu_{\mathrm{v}}}{K_{\mathrm{v}}}+\psi_{1} L_{0}\right\}-\psi_{1}\left(1-\psi_{1}\right) L_{0}^{2}} \\
\times\left(\begin{array}{cc}
\psi_{1}\left(\frac{\mu_{\mathrm{v}}}{K_{\mathrm{v}}}+\psi_{1} L_{0}\right) & \psi_{\mathrm{l}}\left(1-\psi_{1}\right) L_{0} \\
\psi_{\mathrm{l}}\left(1-\psi_{1}\right) L_{0} & \left(1-\psi_{1}\right)\left(\frac{\mu_{1}}{K_{\mathrm{l}}}+\left(1-\psi_{1}\right) L_{0}\right)
\end{array}\right),(13)
\end{gathered}
$$

and $\mu_{\text {eff }}$ is the effective viscosity of the two-phase fluid (scalar). The difference between the conventional equations (10) and (11) and our equations (12) is now clear. We prefer using $K_{1}, K_{\mathrm{v}}$ and $L_{0}$ to using relative permeabilities because of physical simplicity. The permeabilities $K_{1}$ and $K_{\mathrm{v}}$ represent the frictions only between the liquid phase and wall rock, and the vapor phase and wall rock, respectively, and $L_{0}$ represents the friction between the liquid and vapor phases. On the other hand, relative permeabilities include various effects, and their physical meanings are obscure. Moreover, the relative permeability formulation (10) and (11) violates the law of action and reaction between the liquid and vapor phases, since the non-diagonal terms of $\tilde{K}$ should be nonzero as in equation (13) due to the force between the two phases. In addition, the relative permeability formulation is inconvenient for theoretical analyses; the limit of no relative velocity cannot be expressed easily under the action of gravity, although the limit may not be important in realistic situations. Thus we use equations (8) and (9), which are based on the force balance between the two phases, instead of equations (10) and (11).

We further simplify equations (8) and (9). First, we assume that the permeabilities and viscosities are constant and the same for the liquid and vapor, namely, $K_{1}=K_{\mathrm{v}} \equiv K_{0}$ and $\mu_{1}=\mu_{\mathrm{v}} \equiv \mu_{0}$. The approximations are adopted since we intend to make the system simple. The constant permeability means that the resistance between the liquid (vapor) and the wall is proportional to the liquid (vapor) fraction. In addition, the same permeabilities for the two phases mean that their mobilities are the same. The assumption of constant viscosities is justified because we mainly focus on the relative liquid-vapor separation which occurs in a narrow zone as we shall see in Section 4. Moreover, the phase separation occurs around the critical point of the $\mathrm{NaCl}-\mathrm{H}_{2} \mathrm{O}$ system, where the differences of material properties between the two phases are small. An effect of variable viscosity will be discussed in Section 6.1. With these assumptions, we can rewrite equations (8) and (9) to obtain the expressions for the center-of-mass velocity $v$, and the relative velocity $\boldsymbol{v}_{\mathrm{l}}-\boldsymbol{v}_{\mathrm{v}}$ as

$$
\begin{aligned}
& \boldsymbol{v}=-\frac{1}{\phi} \frac{K_{0}}{\mu_{0}} \\
& \cdot\left\{\boldsymbol{\nabla} p-\rho_{\mathrm{f}}\left[1-\omega_{\mathrm{l}}\left(1-\omega_{\mathrm{l}}\right)\left(\rho_{\mathrm{l}}-\rho_{\mathrm{v}}\right)\left(\frac{1}{\rho_{\mathrm{l}}}-\frac{1}{\rho_{\mathrm{v}}}\right) \frac{1}{L_{0} \frac{K_{0}}{\mu_{0}}+1}\right] \boldsymbol{g}\right\},
\end{aligned}
$$

$$
\boldsymbol{v}_{1}-\boldsymbol{v}_{\mathrm{v}}=\frac{1}{\phi} \frac{\rho_{\mathrm{l}}-\rho_{\mathrm{v}}}{\left(L_{0}+\frac{\mu_{0}}{K_{0}}\right)} \boldsymbol{g} .
$$

Second, we assume that

$$
L_{0} \frac{K_{0}}{\mu_{0}} \gg \max \left\{-\omega_{\mathrm{l}}\left(1-\omega_{\mathrm{l}}\right)\left(\rho_{\mathrm{l}}-\rho_{\mathrm{v}}\right)\left(\frac{1}{\rho_{\mathrm{l}}}-\frac{1}{\rho_{\mathrm{v}}}\right), 1\right\} \text {, }
$$

which signifies that the second term in the square bracket in equation (14) is much smaller than the first term (e.g. more than an order of magnitude), and the first term of the denominator of the right hand side of equation (15) is much larger than the second term. This condition signifies that 
the liquid-vapor friction is larger than the wall frictions. As we shall see later in Section 4, we confirm that all of the calculations satisfy the condition (16). This approximation yields

$$
\begin{gathered}
\boldsymbol{v}=-\frac{1}{\phi} \frac{K_{0}}{\mu_{0}}\left(\boldsymbol{\nabla} p-\rho_{\mathrm{f}} \boldsymbol{g}\right), \\
\boldsymbol{v}_{\mathrm{l}}-\boldsymbol{v}_{\mathrm{v}}=\frac{1}{\phi} \frac{\rho_{\mathrm{l}}-\rho_{\mathrm{v}}}{L_{0}} \boldsymbol{g} .
\end{gathered}
$$

We use these equations in our numerical simulations. Here the center-of-mass velocity and the relative velocity are clearly separated, and the separation enables us to interpret the numerical results in a simple way as we shall see later.

Since the density in the buoyancy terms is essential in this problem, we use accurate densities for $\rho_{\mathrm{f}}, \rho_{\mathrm{l}}$, and $\rho_{\mathrm{v}}$ in equations (17) and (18). They are derived from the equation of state of Pitzer and his co-workers as described in Section 2.1.6. The expression for $L_{0}$ is discussed in Section 2.1.5.

2.1.3 Energy conservation The energy conservation equations consist of three equations, because heat is transferred through the rock matrix as well as the vapor and liquid phases. We assume that the rock matrix does not deform. The energy conservation equation for the liquid phase is written as

$$
\begin{aligned}
T \frac{\partial}{\partial t}\left(\phi \psi_{1} \rho_{1} s_{1}\right)+T \boldsymbol{\nabla} & \cdot\left(\phi \psi_{1} \rho_{1} s_{1} v_{1}\right)+\nabla \cdot\left(\phi \psi_{1} J_{q, 1}\right) \\
& =\Gamma_{e,(\mathrm{v} \rightarrow 1)}+\Gamma_{e,(\mathrm{R} \rightarrow \mathrm{l})}+\Phi_{1},
\end{aligned}
$$

the equation for the vapor phase is written as

$$
\begin{aligned}
& T \frac{\partial}{\partial t}\left\{\phi\left(1-\psi_{1}\right) \rho_{\mathrm{v}} s_{\mathrm{v}}\right\}+T \boldsymbol{\nabla} \cdot\left[\phi\left(1-\psi_{1}\right) \rho_{\mathrm{v}} s_{\mathrm{v}} \boldsymbol{v}_{\mathrm{v}}\right] \\
& +\boldsymbol{\nabla} \cdot\left[\phi\left(1-\psi_{1}\right) \boldsymbol{J}_{q, \mathrm{v}}\right]=-\Gamma_{e,(\mathrm{v} \rightarrow \mathrm{l})}+\Gamma_{e,(\mathrm{R} \rightarrow \mathrm{v})}+\Phi_{\mathrm{v}}
\end{aligned}
$$

and the equation for the rock matrix (solid phase) is written as

$T \frac{\partial}{\partial t}\left\{(1-\phi) \rho_{\mathrm{R}} s_{\mathrm{R}}\right\}+\nabla \cdot\left[(1-\phi) \boldsymbol{J}_{q, \mathrm{R}}\right]=-\Gamma_{e,(\mathrm{R} \rightarrow \mathrm{l})}-\Gamma_{e,(\mathrm{R} \rightarrow \mathrm{v})}$,

where $s_{i}(i=1, \mathrm{v}, \mathrm{R})$ is the specific entropy, $\boldsymbol{J}_{q, \mathrm{i}}(i=1, \mathrm{v}, \mathrm{R})$ is the heat flux, $\Gamma_{e,(i \rightarrow j)}(i, j=1, \mathrm{v}, \mathrm{R}, i \neq j)$ is the energy transfer from the $i$-th phase to the $j$-th phase, and $\Phi_{i}(i=1, \mathrm{v})$ is the viscous dissipation for each phase. Here the subscripts $1, \mathrm{v}$, and $\mathrm{R}$ denote the liquid phase, the vapor phase, and the rock matrix, respectively. In these equations, we assume that the temperatures of the three phases are the same. Note that the advection term does not appear in the equation for the rock matrix, because the rock matrix does not move or deform.

To use thermodynamic relationships, we represent the liquid and vapor equations in terms of the Lagrangian derivative of the entropy. The Lagrangian derivative is defined as

$$
\frac{D_{i}}{D t} \equiv \frac{\partial}{\partial t}+\boldsymbol{v}_{i} \cdot \nabla, \quad(i=1, \mathrm{v}) .
$$

By using the mass conservation equations (1) and (2), the energy equations for the liquid and vapor phases (19) and
(20) are modified as

$$
\begin{aligned}
& T \phi \psi_{1} \rho_{1} \frac{D_{1} s_{1}}{D t}+\nabla \cdot\left[\phi \psi_{1} J_{q, 1}\right] \\
& =-T s_{1} \Gamma+\Gamma_{e,(\mathrm{v} \rightarrow 1)}+\Gamma_{e,(\mathrm{R} \rightarrow \mathrm{l})}+\Phi_{1}, \\
& T \phi\left(1-\psi_{1}\right) \rho_{\mathrm{v}} \frac{D_{\mathrm{v}} s_{\mathrm{v}}}{D t}+\nabla \cdot\left[\phi\left(1-\psi_{1}\right) J_{q, \mathrm{v}}\right] \\
& \quad=T s_{\mathrm{v}} \Gamma-\Gamma_{e,(\mathrm{v} \rightarrow \mathrm{l})}+\Gamma_{e,(\mathrm{R} \rightarrow \mathrm{v})}+\Phi_{\mathrm{v}},
\end{aligned}
$$

where $\Gamma$ denotes the rate of phase change from vapor to liquid, and appears in equations (1) and (2). We also rewrite the equation for the solid phase in a similar way. If we assume that the porosity and the rock density are constant, we have

$(1-\phi) \rho_{\mathrm{R}} \frac{\partial s_{\mathrm{R}}}{\partial t}+\boldsymbol{\nabla} \cdot\left[(1-\phi) \boldsymbol{J}_{q, \mathrm{R}}\right]=-\Gamma_{e,(\mathrm{R} \rightarrow \mathrm{l})}-\Gamma_{e,(\mathrm{R} \rightarrow \mathrm{v})}$.

We use the thermodynamic relationships

$$
\rho_{i} T \frac{D_{i} s_{i}}{D t}=\rho_{i} c_{p, i} \frac{D_{i} T}{D t}-\alpha_{i} T \frac{D_{i} p}{D t}, \quad(i=1, \mathrm{v})
$$

on equations (23) and (24), and

$$
\rho_{\mathrm{R}} T \frac{\partial s_{\mathrm{R}}}{\partial t}=\rho_{\mathrm{R}} c_{p, \mathrm{R}} \frac{\partial T}{\partial t}-\alpha_{\mathrm{R}} T \frac{\partial p}{\partial t},
$$

on equation (25), to change the dependent variable from the entropy to the temperature and pressure. Here $\alpha_{i} \quad(i=$ $1, \mathrm{v}, \mathrm{R})$ is the coefficient of thermal expansion for each phase, and $c_{p, i}(i=1, \mathrm{v}, \mathrm{R})$ is the isobaric specific heat capacity for each phase. The equations become

$$
\begin{gathered}
\phi \psi_{1}\left(\rho_{1} c_{p, 1} \frac{D_{1} T}{D t}-\alpha_{1} T \frac{D_{1} p}{D t}\right)+\nabla \cdot\left[\phi \psi_{\mathrm{l}} J_{q, 1}\right] \\
=-T s_{1} \Gamma+\Gamma_{e,(\mathrm{v} \rightarrow \mathrm{l})}+\Gamma_{e,(\mathrm{R} \rightarrow \mathrm{l})}+\Phi_{1}
\end{gathered}
$$

$$
\begin{gathered}
\phi\left(1-\psi_{\mathrm{l}}\right)\left(\rho_{\mathrm{v}} c_{p, \mathrm{v}} \frac{D_{\mathrm{v}} T}{D t}-\alpha_{\mathrm{v}} T \frac{D_{\mathrm{v}} p}{D t}\right)+\boldsymbol{\nabla} \cdot\left[\phi\left(1-\psi_{\mathrm{l}}\right) \boldsymbol{J}_{q, \mathrm{v}}\right] \\
=T s_{\mathrm{v}} \Gamma-\Gamma_{e,(\mathrm{v} \rightarrow \mathrm{l})}+\Gamma_{e,(\mathrm{R} \rightarrow \mathrm{v})}+\Phi_{\mathrm{v}}
\end{gathered}
$$

and

$$
\begin{array}{r}
(1-\phi)\left(\rho_{R} c_{p, \mathrm{R}} \frac{\partial T}{\partial t}-\alpha_{R} T \frac{\partial p}{\partial t}\right)+\nabla \cdot\left[\phi \boldsymbol{J}_{q, \mathrm{R}}\right] \\
=-\Gamma_{e,(\mathrm{R} \rightarrow \mathrm{v})}-\Gamma_{e,(\mathrm{R} \rightarrow \mathrm{l})} .
\end{array}
$$

Neglecting the adiabatic cooling and heating, and viscous dissipation, and assuming Fourier's law which expresses that the heat flux is proportional to the temperature gradient,

$$
J_{q, i}=-\lambda_{i} \nabla T,(i=1, \mathrm{v}, \mathrm{R}),
$$

we can further simplify these equations. Here $\lambda_{i}(i=1, \mathrm{v}, \mathrm{R})$ is the heat conductivity for each phase. Equations (28), (29), and (30) become

$\phi \psi_{1} \rho_{1} c_{p, 1} \frac{D_{1} T}{D t}=\boldsymbol{\nabla} \cdot\left[\phi \psi_{1} \lambda_{1} \nabla T\right]-T s_{1} \Gamma+\Gamma_{e,(\mathrm{v} \rightarrow \mathrm{l})}+\Gamma_{e,(\mathrm{R} \rightarrow \mathrm{l})}$, 


$$
\begin{aligned}
\phi\left(1-\psi_{1}\right) \rho_{\mathrm{v}} c_{p, \mathrm{v}} \frac{D_{\mathrm{v}} T}{D t}= & \boldsymbol{\nabla} \cdot\left[\phi\left(1-\psi_{1}\right) \lambda_{\mathrm{v}} \nabla T\right] \\
& +T s_{\mathrm{v}} \Gamma-\Gamma_{e,(\mathrm{v} \rightarrow \mathrm{l})}+\Gamma_{e,(\mathrm{R} \rightarrow \mathrm{v})},
\end{aligned}
$$

$(1-\phi) \rho_{\mathrm{R}} c_{p, \mathrm{R}} \frac{\partial T}{\partial t}=\nabla \cdot\left[\phi \lambda_{\mathrm{R}} \nabla T\right]-\Gamma_{e,(\mathrm{R} \rightarrow \mathrm{v})}-\Gamma_{e,(\mathrm{R} \rightarrow \mathrm{l})}$.

We neglected adiabatic cooling and heating in the energy conservation equations, because their effect on temperature is $10^{\circ} \mathrm{C}$ at most (e.g. Lowell et al., 1995). We also neglect viscous heating, because the kinetic energy is nearly zero for the permeable flow.

We add equations (32), (33), and (34) to obtain the mixture equation as

$$
\begin{aligned}
\left\{\phi \rho _ { \mathrm { f } } \left[\omega_{\mathrm{l}} c_{p, 1}+\right.\right. & \left.\left.\left(1-\omega_{\mathrm{l}}\right) c_{p, \mathrm{v}}\right]+(1-\phi) \rho_{\mathrm{R}} c_{p, \mathrm{R}}\right\} \frac{\partial T}{\partial t} \\
+ & \phi \rho_{\mathrm{f}}\left\{\left[\omega_{1} c_{p, 1}+\left(1-\omega_{1}\right) c_{p, \mathrm{v}}\right] \boldsymbol{v}\right. \\
& \left.\quad+\omega_{\mathrm{l}}\left(1-\omega_{1}\right) \cdot\left(c_{p, 1}-c_{p, \mathrm{v}}\right)\left(v_{1}-\boldsymbol{v}_{\mathrm{v}}\right)\right\} \cdot \boldsymbol{\nabla} T \\
= & \boldsymbol{\nabla} \cdot\left\{\phi\left[\psi_{1} \lambda_{1}+\left(1-\psi_{1}\right) \lambda_{\mathrm{v}}\right]+(1-\phi) \lambda_{\mathrm{R}}\right\} \boldsymbol{\nabla} T \\
- & T \Delta s \Gamma
\end{aligned}
$$

where $\Delta s=s_{1}-s_{\mathrm{V}}$ is the entropy difference between the liquid and vapor phases. Here the last term of the right-hand side represents the latent heat of phase change. When the chemical equilibrium is maintained, the entropy difference may be replaced by the enthalpy difference as, $\Delta s=\Delta h / T$.

With the Boussinesq approximation, assuming that the porosity is constant, and that the heat capacities and the heat conductivities are constant and the same $\left(c_{\mathrm{p}, 1}=c_{\mathrm{p}, \mathrm{v}}=\right.$ $\left.c_{\mathrm{p}, \mathrm{R}} \equiv c_{\mathrm{p}, 0}, \lambda_{\mathrm{l}}=\lambda_{\mathrm{v}}=\lambda_{\mathrm{R}} \equiv \lambda_{0}\right)$, and neglecting the latent heat $(\Delta s=0)$, we rewrite the mixture equation (35) as

$$
\frac{\partial T}{\partial t}+\phi v \cdot \nabla T=\kappa \nabla^{2} T,
$$

where $\kappa=\lambda_{0} /\left(\rho_{0} c_{\mathrm{p}, 0}\right)$ is the thermal diffusivity. As a result of these simplifications, the energy equation (36) does not depend on the relative velocity between the two phases. We assume that the heat capacities are constant and we neglect the latent heat, because we intend to make the system simple, in accordance with the assumption of constant viscosities in the momentum conservation equations. The adequacy of these assumptions is discussed later in Section 6.4.

We assume that the heat conductivity is constant, because the heat conduction mostly occurs through the rock when the porosity is small, and the heat conductivity of the rock does not vary much over the temperature and pressure ranges of hydrothermal systems, though the heat conductivity of water varies by about an order of magnitude with temperature and pressure (Haar et al., 1984).

2.1.4 NaCl conservation $\mathrm{The} \mathrm{NaCl}$ conservation for the liquid phase is written as

$$
\frac{\partial}{\partial t}\left(\phi \psi_{1} \rho_{1} C_{1}\right)+\nabla \cdot\left(\phi \psi_{1} \rho_{1} C_{1} v_{1}\right)+\nabla \cdot\left(\phi \psi_{1} \rho_{1} J_{D, 1}\right)=\Gamma_{c},
$$

and the equation for the vapor phase is written as

$$
\begin{array}{r}
\frac{\partial}{\partial t}\left[\phi\left(1-\psi_{1}\right) \rho_{\mathrm{v}} C_{\mathrm{v}}\right]+\boldsymbol{\nabla} \cdot\left[\left(1-\psi_{1}\right) \rho_{\mathrm{v}} C_{\mathrm{v}} \boldsymbol{v}_{\mathrm{v}}\right] \\
+\boldsymbol{\nabla} \cdot\left[\phi\left(1-\psi_{1}\right) \rho_{\mathrm{v}} \boldsymbol{J}_{D, \mathrm{v}}\right]=-\Gamma_{c}
\end{array}
$$

where $C_{1}$ is the $\mathrm{NaCl}$ concentration in the liquid phase, $C_{\mathrm{v}}$ is the $\mathrm{NaCl}$ concentration in the vapor phase, $\boldsymbol{J}_{D, 1}$ is the diffusive flux of $\mathrm{NaCl}$ in the liquid phase, $\boldsymbol{J}_{D, \mathrm{v}}$ is that in the vapor phase, and $\Gamma_{c}$ represents the transfer of $\mathrm{NaCl}$ from vapor to liquid. Note that the $\mathrm{NaCl}$ concentration $C_{i}$ 's are defined as the mass of $\mathrm{NaCl}$ per unit mass of a phase $i$ ( $i=$ $1, \mathrm{v})$.

The mixture equation is obtained by adding equations (37) and (38) as

$$
\begin{array}{r}
\frac{\partial}{\partial t}\left(\phi \rho_{\mathrm{f}} C\right)+\boldsymbol{\nabla} \cdot \phi \rho_{\mathrm{f}}\left[C \boldsymbol{v}+\omega_{\mathrm{l}}\left(1-\omega_{\mathrm{l}}\right)\left(C_{1}-C_{\mathrm{v}}\right)\left(\boldsymbol{v}_{1}-\boldsymbol{v}_{\mathrm{v}}\right)\right] \\
+\boldsymbol{\nabla} \cdot\left(\phi \rho_{\mathrm{f}} \boldsymbol{J}_{D}\right)=0
\end{array}
$$

where the mean $\mathrm{NaCl}$ concentration of the two-phase fluid $C$ is defined as

$$
C=\omega_{1} C_{1}+\left(1-\omega_{1}\right) C_{\mathrm{v}},
$$

and the total flux of the material diffusion $\boldsymbol{J}_{D}$ is defined as

$$
\boldsymbol{J}_{D}=\omega_{\mathrm{l}} \boldsymbol{J}_{D, 1}+\left(1-\omega_{\mathrm{l}}\right) \boldsymbol{J}_{D, \mathrm{v}} .
$$

We assume Fick's law for the diffusive flux,

$$
\boldsymbol{J}_{D}=-D \nabla C,
$$

where $D$ is the coefficient of material diffusion. This equation signifies that the diffusive flux is proportional to the gradient of the mean $\mathrm{NaCl}$ concentration. With the Boussinesq approximation, constant porosity, and Fick's law, we can rewrite equation (39) as

$\phi \frac{\partial C}{\partial t}+\nabla \cdot \phi\left[C v+\omega_{1}\left(1-\omega_{1}\right)\left(C_{1}-C_{\mathrm{v}}\right)\left(v_{1}-v_{\mathrm{v}}\right)\right]=\phi D \nabla^{2} C$.

We assume that chemical equilibrium is realized between the two phases so that we directly obtain the $\mathrm{NaCl}$ concentrations of the two phases from the temperature and pressure by using the phase diagram. The mass fraction of the liquid phase $\omega_{1}$ is related to the $\mathrm{NaCl}$ concentrations by the lever rule as

$$
\omega_{1}=\frac{C-C_{\mathrm{v}}}{C_{1}-C_{\mathrm{v}}} .
$$

The $\mathrm{NaCl}$ concentrations of the liquid and vapor phases are derived from the phase diagram of Pitzer and his co-workers as we explain in Section 2.1.6.

2.1.5 Relative velocity In Section 2.1.2, we have derived the approximation that the relative velocity between the liquid and vapor phases is proportional to the density difference. The next step is to determine the friction coefficient $L_{0}$. We assume that it has the form similar to Stokes' law, namely, it is proportional to the fluid viscosity. This assumption is applicable when the Reynolds number based on the typical size of the geometry of the two-phase boundaries is less than the order of unity. Then the friction constant becomes

$$
L_{0}=\frac{1}{\phi} \frac{\mu_{0}}{k} .
$$

Here $k$ is a coefficient which represents the magnitude of the friction between the two phases. It has a dimension of a square of length, and may be written as

$$
k=f a^{2},
$$




\section{$\operatorname{Density}\left(\mathrm{kg} / \mathrm{m}^{3}\right)$}

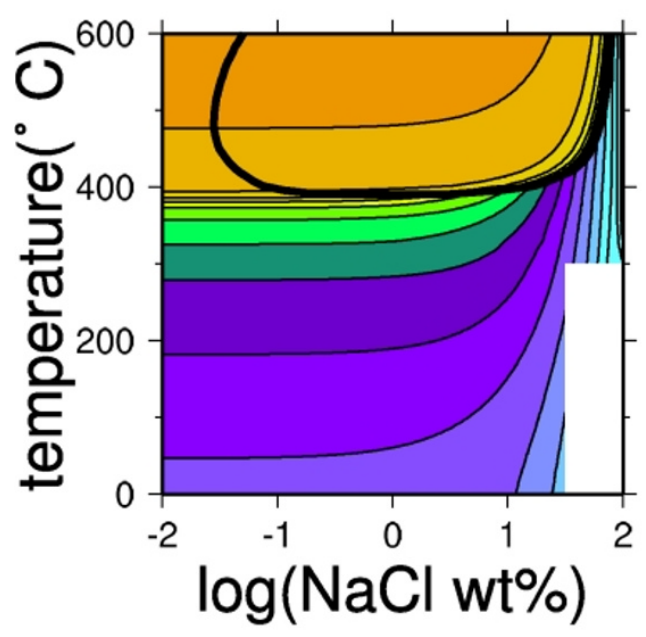

a) $\mathrm{P}=25(\mathrm{MPa})$
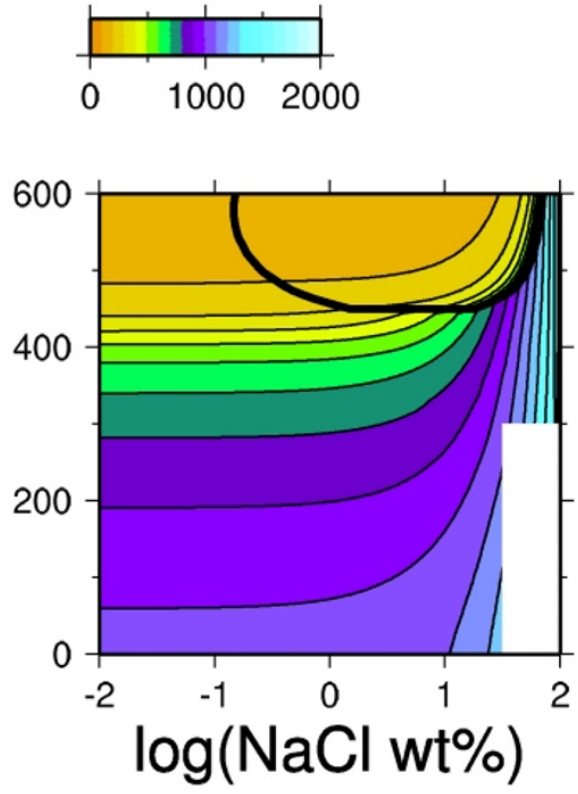

b) $\mathrm{P}=40(\mathrm{MPa})$

Fig. 2. a) Density of $\mathrm{NaCl}-\mathrm{H}_{2} \mathrm{O}$ two-component fluid is shown together with liquid-vapor co-existing curves in the NaCl concentration and temperature space for a pressure of $25 \mathrm{MPa}$, based on the theory of Pitzer and his co-workers (Pitzer et al., 1984; Anderko and Pitzer, 1993). The vertical axis is the temperature in Celsius degree, and the horizontal axis is the logarithm of the $\mathrm{NaCl}$ concentration in wt\%. The thick solid curves are the two-phase coexisting curves, which are the same as those in Fig. 1. In the two-phase region, the mean density is calculated by the volume-weighted average of the two phases (see equation (5)). The blank area at low temperatures and high salinities is out of the range for which the equation of state is applicable. b) Same as a) but the pressure is $40 \mathrm{MPa}$.

where $f$ is a non-dimensional factor which depends on the mode of relative separation, and $a$ is a typical length scale of two-phase boundaries. The choice of $a$ is arbitrary. When equation (45) holds, the relative velocity between the two phases (18) is represented as

$$
\boldsymbol{v}_{1}-\boldsymbol{v}_{\mathrm{v}}=k \frac{\rho_{\mathrm{l}}-\rho_{\mathrm{v}}}{\mu_{0}} \boldsymbol{g}
$$

In this study, we treat the coefficient $k$ as a constant parameter, without specifying the mode of relative separation (see Section 3). This is because we intend to make the system simple, although in reality $f$ is a complex function of the liquid volume fraction and the geometry of two-phase boundaries. An example of the factor $f$ is given by classical Stokes' law (e.g. Batchelor, 1967), when one phase occupies a small fraction, and forms spherical droplets or bubbles of the same size. If we take $a$ as the radius of the spherical bodies, $f$ is written as

$$
f=\frac{2}{3} \frac{\mu_{0}}{\mu_{1}} \frac{\mu_{1}+\mu_{2}}{2 \mu_{1}+3 \mu_{2}},
$$

where $\mu_{1}$ is the viscosity of the phase which occupies a major fraction, and $\mu_{2}$ is the viscosity of the phase which forms bubbles or droplets. When the viscosities of the two phases are the same,

$$
f=\frac{4}{15} .
$$

2.1.6 Equation of state The equation of state (density as a function of pressure, temperature, and salinity) and the liquid-vapor coexisting curve of the $\mathrm{NaCl}-\mathrm{H}_{2} \mathrm{O}$ system are investigated in detail by Pitzer and his co-workers (Pitzer et al., 1984; Anderko and Pitzer, 1993). We use their semiempirical equations to obtain the density and liquid-vapor coexisting conditions. We use Anderko and Pitzer's (1993) model for temperatures higher than $300^{\circ} \mathrm{C}$, Pitzer et al.'s (1984) for temperatures lower than $300^{\circ} \mathrm{C}$.

Figure 1 shows the two-phase coexisting curves at various pressures. The co-existing curves show the $\mathrm{NaCl}$ concentrations of the liquid-like and vapor-like phases in equilibrium at each temperature and pressure. The curves are U-shaped when the pressure is larger than the critical pressure of pure water. The minimum temperature on a co-existing curve in the $(C, T)$ space is called the critical temperature. If the mean concentration $C$ falls within the two-phase region, the region above the co-existing curve, the fluid separates into liquid-like and vapor-like phases. Seawater separates at a temperature close to the critical one in the pressure range of this study.

Figure 2 shows the densities at pressures of 25 and 40 $\mathrm{MPa}$. The property which is most important for our simulations is the increase in density with increasing temperature along the liquid-like branch of the co-existing curve. As we show later in Section 4, the concentration $C$ runs along the liquid-like branch near the bottom of the calculation area. In this region, the effective thermal expansion becomes negative, because the density increases with temperature due to the increase of $\mathrm{NaCl}$ concentration along the liquid-like branch. The comparison of Figs. 2(a) and 2(b) shows that the pressure dependence of the co-existing curve is more conspicuous than the pressure dependence of the density.

In Figs. 1 and 2, the precipitation of halite is neglected. 


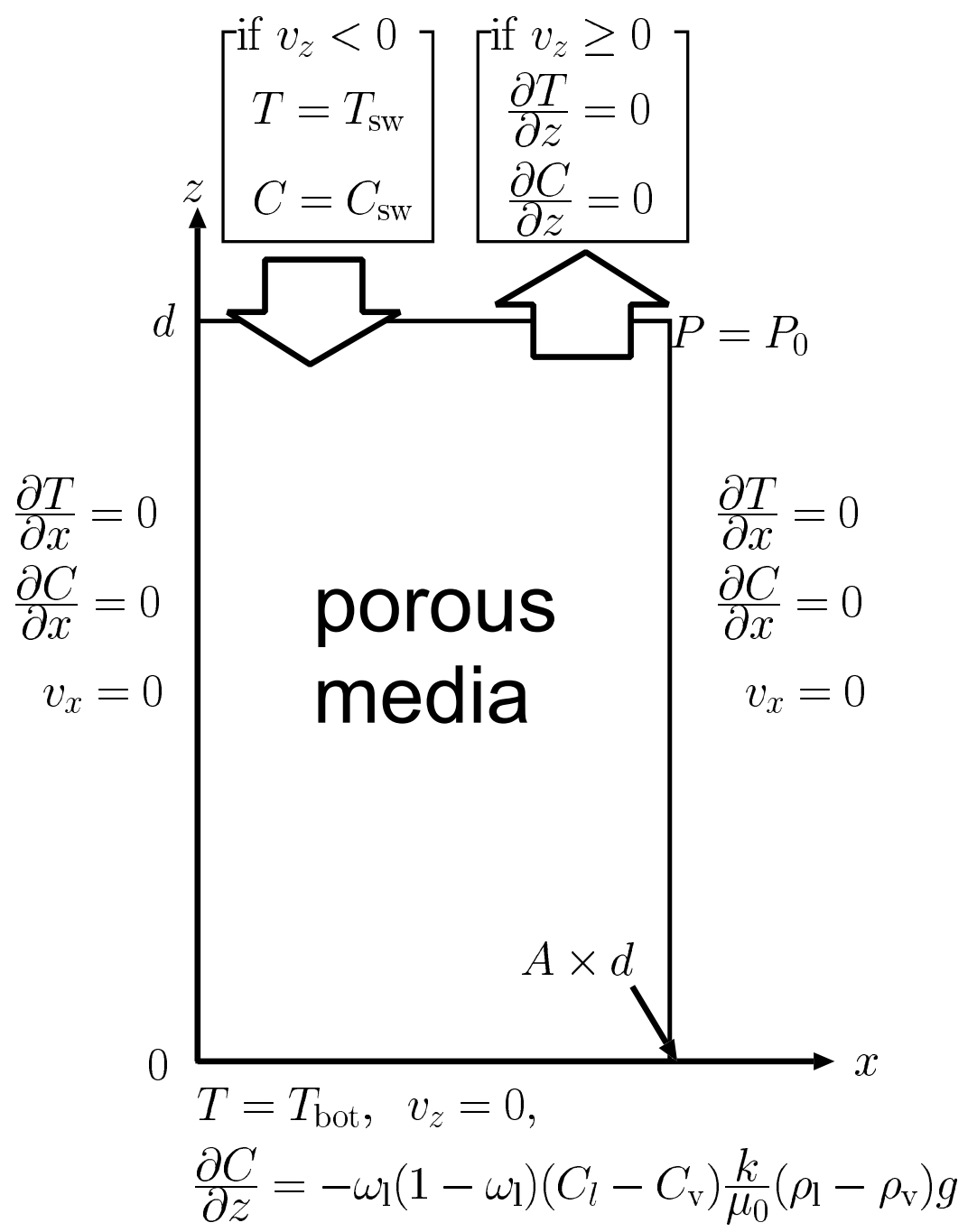

Fig. 3. Summary of the dimensional boundary conditions. Note that the top boundary conditions depend on the direction of the mean flow velocity. The height of the calculation area is denoted by $d$, and $A$ is its aspect ratio, so that $A d$ is its width.

In Section 4, we shall confirm that the fluid does not enter the halite precipitation region on the phase diagram in the calculations of this study.

We use two kinds of pressures, namely, the hydrostatic pressure $p_{e}$, and the dynamic pressure, $p$. The hydrostatic pressure $p_{e}$ is used for calculating the equation of state $\left(\rho_{\mathrm{l}}\right.$, $\rho_{\mathrm{v}}$, and $\left.\rho_{\mathrm{f}}\right)$ and the two-phase co-existing curve $\left(C_{1}\right.$ and $\left.C_{\mathrm{v}}\right)$. The dynamic pressure $p$ is used for calculating the equation of motion (17). The two pressures are independent. The hydrostatic pressure is defined as

$$
p_{e}=\rho_{0} g(d-z)+\rho_{0} g d_{\mathrm{sf}} .
$$

Here $\rho_{0}$ is a typical density of the fluid, $d$ is the height of the calculation area, $d_{\text {sf }}$ is the depth of the seafloor, $g$ is the magnitude of the gravity acceleration, $z$ is the vertical coordinate, taken upward positive, with $z=0$ and $d$ denoting the bottom and top of the calculation area, respectively (Fig. 3). The top is the seafloor. We use $10^{3} \mathrm{~kg} \mathrm{~m}^{-3}$ for $\rho_{0}$, and $10 \mathrm{~m} \mathrm{~s}^{-2}$ for $g$. This approximation (50) is frequently used for calculations of incompressible fluids (e.g. Christensen, 1984). The use of this relation is justified because dynamical effects of pressure on the calculation of material properties are small. Equation (50) shows that there is one-to-one relationship be- tween the pressure and the depth for calculating the material properties. Thereby we can regard the equation of state and two-phase coexisting curve as functions of $(z, T, C)$ instead of $(p, T, C)$. For instance, at the seafloor whose depth $d_{\text {sf }}$ is $2500 \mathrm{~m}$, we use the phase diagram for a pressure of 25 MPa. Similarly, at the base whose depth is $4000 \mathrm{~m}$, which corresponds to $d=1500 \mathrm{~m}$, we use the phase diagram for a pressure of $40 \mathrm{MPa}$.

\subsection{Boundary conditions}

Figure 3 illustrates the boundary conditions we adopt. The top boundary conditions are similar to those of Wilcock (1998), and given as

$$
p=p_{0}, \quad\left\{\begin{array}{l}
T=T_{\mathrm{sw}}, C=C_{\mathrm{sw}},\left(\text { if } v_{z}<0\right) \\
\frac{\partial T}{\partial z}=0, \frac{\partial C}{\partial z}=0,\left(\text { if } v_{z} \geq 0\right)
\end{array} \text { at } z=d,\right.
$$

where the subscript $z$ denotes the vertical component, taken upward positive, $p_{0}$ denotes the dynamic pressure at the seafloor, and $T_{\mathrm{sw}}$ and $C_{\mathrm{sw}}$ are the temperature and $\mathrm{NaCl}$ concentration of seawater, respectively. The fluid which flows into the oceanic crust has the temperature and $\mathrm{NaCl}$ concentration of seawater. The fluid which flows out of the 
system keeps its temperature and $\mathrm{NaCl}$ concentration. The dynamic pressure is constant at the top boundary, where the fluid can flow in or out. The velocity is calculated to satisfy the boundary condition for the dynamic pressure. Note that adding a constant to the dynamic pressure $p$ has no effects on the velocity field, because the dynamic pressure appears only in the pressure gradient term in the equation of motion. We do not use the dynamic pressure $p$ for calculating the equation of state and the liquid-vapor co-existing curves, for which we use the hydrostatic pressure $p_{e}$ (see Section 2.1.6). Thus we may choose an arbitrary value for the dynamic pressure at the top boundary $p_{0}$.

The bottom boundary is impermeable, and its temperature is constant:

$$
T=T_{\text {bot }}, \quad v_{z}=0 \quad \text { at } z=0,
$$

where $T_{\text {bot }}$ is the bottom temperature. The bottom boundary condition for the $\mathrm{NaCl}$ concentration, which is equivalent to the condition of no material flux, is derived from equation (43) as

$$
\frac{\partial C}{\partial z}=\frac{1}{D} \omega_{\mathrm{l}}\left(1-\omega_{1}\right)\left(C_{1}-C_{\mathrm{v}}\right)\left(v_{\mathrm{l}, z}-v_{\mathrm{v}, z}\right) \quad \text { at } z=0 .
$$

The derivation of this equation is as follows. We make use of a thin and planar volume straddling the bottom boundary. The top surface of the volume is in the porous media, and its bottom surface lies in the impermeable rock. Integrating the $\mathrm{NaCl}$ conservation equation (43) over this volume, we have

$$
\left[D \frac{\partial C}{\partial z}\right]_{-}^{+}=\left[C v_{z}\right]_{-}^{+}+\left[\omega_{\mathrm{l}}\left(1-\omega_{1}\right)\left(C_{1}-C_{\mathrm{v}}\right)\left(v_{\mathrm{l}, \mathrm{z}}-v_{\mathrm{v}, \mathrm{z}}\right)\right]_{-}^{+},
$$

where the superscript $(+)$ denotes the top surface of the volume, and the subscript $(-)$ denotes its bottom surface. All the terms which have the subscript $(-)$ vanish, because the bottom boundary is impermeable. The first term of the right hand side vanishes, because $v_{z}=0$ at the boundary. Thus, the boundary condition (54) becomes

$$
\left[D \frac{\partial C}{\partial z}\right]_{z=0+}=\left[\omega_{\mathrm{l}}\left(1-\omega_{1}\right)\left(C_{1}-C_{\mathrm{v}}\right)\left(v_{\mathrm{l}, \mathrm{z}}-v_{\mathrm{v}, \mathrm{z}}\right)\right]_{z=0+}
$$

The right hand side of equation (55) is negative because dense brine sinks to the bottom, and this should be balanced by upward diffusive flux.

The side boundaries are adiabatic, impermeable, and do not allow salt to pass through:

$$
\frac{\partial T}{\partial x}=0, \quad \frac{\partial C}{\partial x}=0, \quad v_{x}=0 \quad \text { at } x=0, A d .
$$

Here the subscript $x$ denotes the horizontal coordinate, and $A$ is the aspect ratio of the calculation region, defined as the ratio of the width to the height.

\subsection{Non-dimensionalization}

Equations (7), (17), (36), (43) and (47) are nondimensionalized with the thickness of the porous media $d$ as the length scale, the thermal conduction time $d^{2} / \kappa$ as the time scale, $\rho_{0}$ as the density scale, $\rho_{0} g K_{0} / \mu_{0}$ as the velocity scale, $\rho_{0} g d$ as the pressure scale, and the temperature difference between the seawater and the bottom boundary $\left(T_{\mathrm{bot}}-T_{\mathrm{sw}}\right)$ as the temperature scale.

The time dependent non-dimensionalized equations are

$$
\begin{gathered}
\boldsymbol{\nabla} \cdot \boldsymbol{v}=0, \\
\phi \boldsymbol{v}=-\nabla p-\rho_{\mathrm{f}} \boldsymbol{e}_{z}, \\
\frac{\partial T}{\partial t}+R a \boldsymbol{\nabla} \cdot(\phi \boldsymbol{v} T)=\nabla^{2} T,
\end{gathered}
$$

$$
\begin{aligned}
& \phi \frac{\partial C}{\partial t}+\operatorname{Ra} \nabla \cdot(\phi v C) \\
& \quad+\phi R a \nabla \cdot\left[\omega_{1}\left(1-\omega_{1}\right)\left(C_{1}-C_{\mathrm{v}}\right)\left(v_{1}-v_{\mathrm{v}}\right)\right]=\frac{\phi}{L e} \nabla^{2} C,
\end{aligned}
$$

$$
\boldsymbol{v}_{1}-\boldsymbol{v}_{\mathrm{v}}=-\Pi\left(\rho_{1}-\rho_{\mathrm{v}}\right) \boldsymbol{e}_{z},
$$

where $\boldsymbol{e}_{z}$ is the unit vector pointing upward. Note that this unit vector is related to the gravitational acceleration $g$ as $\boldsymbol{g}=-g \boldsymbol{e}_{z}$. Here we denote the non-dimensionalized variables with the same symbols as those of the corresponding dimensionalized variables. Note that $\rho_{\mathrm{f}}=\rho_{\mathrm{f}}(z, T, C), \rho_{\mathrm{l}}=$ $\rho_{\mathrm{l}}(z, T), \rho_{\mathrm{v}}=\rho_{\mathrm{v}}(z, T), C_{1}=C_{\mathrm{l}}(z, T)$, and $C_{\mathrm{v}}=C_{\mathrm{v}}(z, T)$, as we described in Section 2.1.6.

The non-dimensional numbers in these equations are defined as

$$
\begin{gathered}
R a=\frac{\rho_{0} g K_{0} d}{\mu_{0} \kappa}, \\
\Pi=\frac{k}{K_{0}},
\end{gathered}
$$

and

$$
\frac{1}{L e}=\frac{D}{\kappa} \text {. }
$$

Here the Rayleigh number $R a$ is the ratio of the time scale of the mean motion to the thermal diffusion time, $\Pi$ is the ratio of the coefficient of the resistance between the two phases to the permeability (resistance between the fluid and the rock, in our definition), and $1 / L e$ is called the inverse Lewis number, which is the ratio of material diffusion to thermal diffusion. The parameter $\Pi$ controls the efficiency of phase separation. The larger the parameter $\Pi$ is, the more effective the phase separation becomes. If $\Pi \ll 1$, the flow becomes similar to simple one-phase convection.

It is to be noted that the definition of the Rayleigh number (62) is different from the conventional one (e.g. Nield and Bejan, 1999). Here, the Rayleigh number is defined as $R a=\rho_{0} g d K / \mu_{0} \kappa$, in which the density is scaled by a typical density $\rho_{0}$, whereas the conventional definition is $R a_{n}=\Delta \rho g d K / \mu_{0} \kappa$, in which the density is scaled by the density difference between the densities at the upper and lower boundaries $\Delta \rho$. We use the former definition, because the densities at the upper and the lower boundaries are not constants in this calculation. For seawater, $\Delta \rho / \rho_{0}$ is of the order of unity, if we assume that $\rho_{0}=10^{3} \mathrm{~kg} \mathrm{~m}^{-3}$ and $\Delta \rho=\rho_{\mathrm{sw}}-\rho_{\mathrm{cr}} \simeq 600 \mathrm{~kg} \mathrm{~m}^{-3}$, with $\rho_{\mathrm{sw}}=1030 \mathrm{~kg} \mathrm{~m}^{-3}$ and $\rho_{\mathrm{cr}}=440 \mathrm{~kg} \mathrm{~m}^{-3}$, where $\rho_{\mathrm{cr}}$ is the density of seawater at the critical point. Hence the magnitudes of our Rayleigh number $R a$ and the conventional Rayleigh number $R a_{n}$ are of the same order, $R a \simeq R a_{n}$. 
Table 1. Parameters used in the calculations, and the results.

\begin{tabular}{ccc|ccccc}
\hline$R a$ & $\Pi^{\mathrm{a}}$ & $1 / L e$ & $N u$ & $\begin{array}{c}C_{\min } \\
\mathrm{wt} \%\end{array}$ & $\begin{array}{c}C_{\max } \\
\mathrm{wt} \%\end{array}$ & $\begin{array}{c}T_{\max } \\
{ }^{\circ} \mathrm{C}\end{array}$ & $\delta^{\mathrm{b}}$ \\
\hline 100 & 0 & - & 3.25 & - & - & 299. & - \\
100 & 5 & 1 & 1.81 & 2.54 & 4.42 & 215. & 0.12 \\
100 & 10 & 1 & 1.45 & 2.99 & 3.59 & 183. & 0.17 \\
100 & 20 & 1 & 1.37 & 3.15 & 3.30 & 175. & 0.19 \\
\hline
\end{tabular}

${ }^{a} \Pi=0$ corresponds to thermal convection of a one-phase fluid.

${ }^{b} \delta$ : mean thickness of the stagnant layer.

Equations (60) and (61) are merged into

$$
\begin{aligned}
& \phi \frac{\partial C}{\partial t}+R a \nabla \cdot(\phi v C) \\
& -\phi \Pi R a \frac{\partial}{\partial z} \frac{\left(C-C_{\mathrm{v}}\right)\left(C_{1}-C\right)}{C_{1}-C_{\mathrm{v}}}\left(\rho_{1}-\rho_{\mathrm{v}}\right)=\frac{\phi}{L e} \nabla^{2} C,
\end{aligned}
$$

by using the relation (44). We solve equations (57), (58), (59), and (65), together with the equation of state and the liquid-vapor co-existing curve. Equations (58), (61) and (65) are applicable when the condition

$$
\frac{1}{\Pi} \gg \phi \max \left\{\left[-\omega_{1}\left(1-\omega_{1}\right)\left(\rho_{1}-\rho_{\mathrm{v}}\right)\left(\frac{1}{\rho_{\mathrm{l}}}-\frac{1}{\rho_{\mathrm{v}}}\right)\right], 1\right\},
$$

is satisfied. It is the non-dimensionalized form of equation (16). We shall later confirm in Section 4 that the condition (66) is satisfied in our results.

The non-dimensionalized boundary conditions are

$$
p=0, \quad\left\{\begin{array}{l}
T=T_{\mathrm{sw}}, C=C_{\mathrm{sw}},\left(\text { if } v_{z}<0\right) \\
\frac{\partial T}{\partial z}=0, \frac{\partial C}{\partial z}=0,\left(\text { if } v_{z} \geq 0\right)
\end{array} \quad \text { at } z=1,\right.
$$

$$
\begin{array}{r}
T=T_{\mathrm{bot}}, \quad v_{z}=0, \quad \frac{\partial C}{\partial z}= \\
-\left(\rho_{1}-\rho_{\mathrm{v}}\right) \quad \text { at } z=0
\end{array}
$$

and

$$
\frac{\partial T}{\partial x}=0, \quad \frac{\partial C}{\partial x}=0, \quad v_{x}=0 \quad \text { at } x=0, A .
$$

Although all the parameters and constants are nondimensionalized in the numerical calculations, we sometimes use dimensional values in the following sections for convenience of explanation.

\section{Numerical Calculations}

We calculate steady flow in a two-dimensional rectangular region. The vertical extent of the calculation area is from the seafloor to the brittle-ductile front. The depth of the seafloor from the sea surface is set at $2500 \mathrm{~m}$, which is a typical depth of hydrothermal systems (e.g. Fornari and Embley, 1995). The depth of the brittle-ductile front from the sea surface is set at $4000 \mathrm{~m}$, which is taken from observations of the depth of seismic reflection at ridge axes (e.g. Sinton and Detrick,
1992). In the non-dimensional vertical coordinate, $z=1$ represents the seafloor, and $z=0$ represents the base of the calculation region.

The width of the calculation area is $750 \mathrm{~m}$, which corresponds to the aspect ratio of $A=0.5$. This value is chosen so that one circulation cell should appear. When $A=1.0$ (square cell), two circulation cells appear in the steady state if $R a=100$ and $\Pi=10$. We argue that one circulation cell would be enough for investigating steady state structure, because mid-ocean ridge hydrothermal circulation would basically have symmetric two-cell structure with an upwelling located around the crest. We fix the aspect ratio $A$ in spite of its dependence on various parameters (e.g. Nield and Bejan, 1999), because our calculations are carried out over relatively narrow range of parameters as we explain later in this section.

The porosity of the rock matrix is set at a constant value 0.01 , which is taken from observations of sheeted dike sequences in the oceanic crust (Nehlig and Juteau, 1988; Becker, 1989). The pore space is filled with $\mathrm{NaCl}-\mathrm{H}_{2} \mathrm{O}$ twocomponent fluids.

The value of $T_{\mathrm{sw}}, C_{\mathrm{sw}}$, and $T_{\mathrm{bot}}$ for the boundary conditions (67) and (68) are set as follows. The temperature of seawater $T_{\mathrm{sw}}$ is $0^{\circ} \mathrm{C}$ in a dimensional value and 0 in a nondimensional value. The $\mathrm{NaCl}$ concentration of seawater $C_{\mathrm{sw}}$ is $3.2 \mathrm{wt} \%$ (non-dimensional). The bottom temperature $T_{\mathrm{bot}}$, the temperature of brittle-ductile transition of rocks, is set at $600^{\circ} \mathrm{C}$ in a dimensional value and 1 in a non-dimensional value. This value is within the range of the temperatures at the base of hydrothermal circulation, $500-800^{\circ} \mathrm{C}$, inferred from geological observations (Mével and Cannat, 1991).

The hydrostatic pressure $p_{e}$ is defined as

$$
\left(\frac{p_{e}}{\mathrm{MPa}}\right)=40-15 z
$$

where $z$ is the non-dimensional height. It is used to calculate the equation of state $\left(\rho_{\mathrm{l}}\right.$ and $\left.\rho_{\mathrm{v}}\right)$ and the liquid-vapor coexisting curve $\left(C_{1}\right.$ and $\left.C_{\mathrm{v}}\right)$. Note again that the hydrostatic pressure $p_{e}$ and the dynamic pressure $p$, used for calculating the equation of motion (58), are independent as we explained in Section 2.1.6.

The non-dimensional parameters used for calculating dynamics are shown in Table 1 . The most important parameter is $\Pi$, since it controls the efficiency of phase separation. Large $\Pi$ results in effective phase separation, and small $\Pi$ results in a flow similar to one-phase convection. It can therefore be inferred that there will be two flow regimes: a flow with little phase separation and a flow with almost com- 
Table 2. Parameters used for estimating the Rayleigh number.

\begin{tabular}{clll}
\hline symbol & meaning & value & \\
\hline$g$ & gravity acceleration & 10 & $\mathrm{~m} \mathrm{~s}^{-2}$ \\
$d$ & depth of circulation & $\mathrm{m}$ \\
$K$ & permeability & $10^{3}$ & $\mathrm{~m}^{2}$ \\
$v$ & kinematic viscosity of water $^{\mathrm{b}}$ & $10^{-8}-10^{-17}$ & $\mathrm{~m}^{2} \mathrm{~s}^{-1}$ \\
$\kappa$ & thermal diffusivity of rock $^{\mathrm{c}}$ & $10^{-6}$ & $\mathrm{~m}^{2} \mathrm{~s}^{-1}$ \\
& & & \\
$R a$ & Rayleigh number & $10^{0}-10^{9}$ & \\
\hline
\end{tabular}

${ }^{a}$ The depth of circulation $d$ is estimated from observations of the depth of seismic reflection surfaces at ridge axes (Sinton and Detrick, 1992).

${ }^{b}$ The typical kinematic viscosity is taken from Haar et al. (1984).

${ }^{c}$ The thermal diffusivity should be that of rocks, because the heat is transferred mainly through rocks.

plete phase separation. There should be a transition at around some $\Pi$, for which the velocity of relative separation and the mean flow velocity become comparable, and that is where detailed investigation is required. We carry out numerical calculations for a wide range of $\Pi$ to find that the transition between the two flow regimes occurs at around $\Pi=10$ (see results in Section 4). Hence we show results for values from 5 to 20 in the following sections.

The physical meaning of $\Pi$ in terms of texture of rocks can be understood if we write $\Pi$ as

$$
\Pi=\frac{180 f}{\phi}\left(\frac{a}{b}\right)^{2},
$$

by using equation (46) and Kozeny-Carman's relation (e.g. Bear, 1988),

$$
K_{0}=\frac{\phi}{180} b^{2},
$$

where $b$ is a typical width of the cracks. Here the crack width $b$ is related to a typical grain size $r_{\mathrm{m}}$ as $b=r_{\mathrm{m}} \phi /(1-\phi)$. If $\phi=0.01$ and $f=4 / 15$ (see Section 2.1.5), the value $\Pi=10$ is realized when $a / b=0.05$. The phase separation becomes inefficient as $a$ decreases because the drag between the two phases increases. On the other hand, large $a$ results in effective phase separation. In reality, however, clogging will occur when $a$ is comparable to $b$, but this effect is not included in equation (71) because surface tension, which is not explicitly considered in this formulation, is essential for clogging.

We choose the Rayleigh number $R a$ of 100 mainly for a computational reason. The numerical convergence is within $3 \%$ for this Rayleigh number, but we cannot obtain good numerical convergence for larger Rayleigh numbers. This Rayleigh number of 100 is about ten times the critical conventional Rayleigh number (Section 2.3) for simple onephase thermal convection (Nield and Bejan, 1999); the critical conventional Rayleigh number is $\pi^{2}$, when the bottom boundary is impermeable and isothermal, and the top boundary has a constant pressure and a constant conductive heat flux.

The Rayleigh number estimated from physical properties of rocks and seawater can be as small as 1 or as large as $10^{9}$ (Table 2). The uncertainty is mainly due to that of the permeability of the rock. The lowest estimated value of the permeability comes from off-axis drilled cores from various sites. For instance, in the Hole 504B, which is located to the south of the Costa Rica Rift, the permeability is measured to be $10^{-14} \mathrm{~m}^{2}$ for the extrusive basalt, and $10^{-17} \mathrm{~m}^{2}$ for the sheeted dike (Becker, 1989). Similar estimates are reported from other sites (Fisher, 1998). The Rayleigh number becomes $10^{3}$ and 1 for these permeabilities, respectively. The Rayleigh number of 100 , which we use for numerical calculations is similar to the estimate for the extrusive basalt. It corresponds to the permeability $K_{0}$ of $10^{-15} \mathrm{~m}^{2}$. The highest value comes from the mineral-filled cracks in Samail ophiolite, Oman (Nehlig and Juteau, 1988; Nehlig, 1994). The estimated permeabilities range from $10^{-8}$ to $10^{-11} \mathrm{~m}^{2}$ for the sheeted dike sequence. The Rayleigh numbers for these permeabilities range from $10^{6}$ to $10^{9}$. There are no data for extrusive basalts for the ophiolite.

We treat $1 / L e$ as a constant and use the value of 1 . Realistic values of the parameter $1 / L e$ are also uncertain, but $1 /$ Le has a constraint that it should not be much larger than the order of unity. If we take molecular diffusivities, it is less than unity $\left(1-10^{-3}\right.$, depending mainly on temperature; e.g. Lasaga, 1998). If we take effective diffusivities (hydrodynamic dispersion), it should be of the order of unity. We use the value of 1 because the diffusion expressed by equation (42) should be interpreted as hydrodynamic diffusion. Molecular diffusion cannot be expressed in that simple way for two-phase flow.

We use a staggered-grid finite difference method for numerical calculations. We use an evenly spaced numerical grid, with the number of the mesh cells being 128 in the horizontal direction and 256 in the vertical direction. Steady state solutions are obtained by time-marching; we start calculations with a diffusive solution, and proceed until the horizontally averaged $\mathrm{NaCl}$ concentration at $z=1$ (exactly defined by equation (74), explained later in Section 4) and the Nusselt number converge within $10^{-9}$ between two time steps. This condition is sufficient, since the step is about $2 \times 10^{-6}$, which is restricted by thermal conduction, and that the convergence level of $10^{-9}$ means that the quantities converge within $0.5 \%$ in unit nondimensional time.

The non-linear advection terms in the energy equation (59) and $\mathrm{NaCl}$ conservation (65) equation are solved as follows. The center of mass advection terms in both equations are solved with the first-order skew-upwind scheme (Raithby, 1976). This method prevents numerical diffusion normal to 

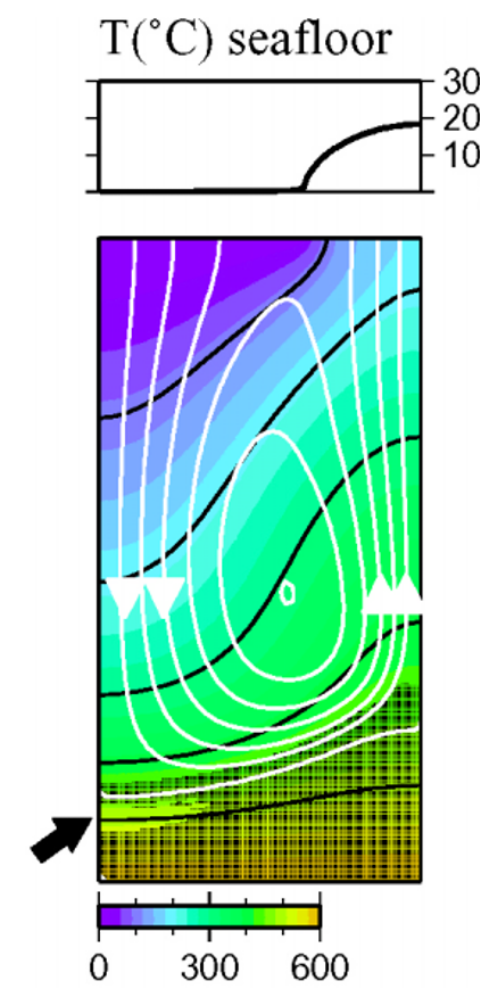

a)Temperature $\left({ }^{\circ} \mathrm{C}\right)$
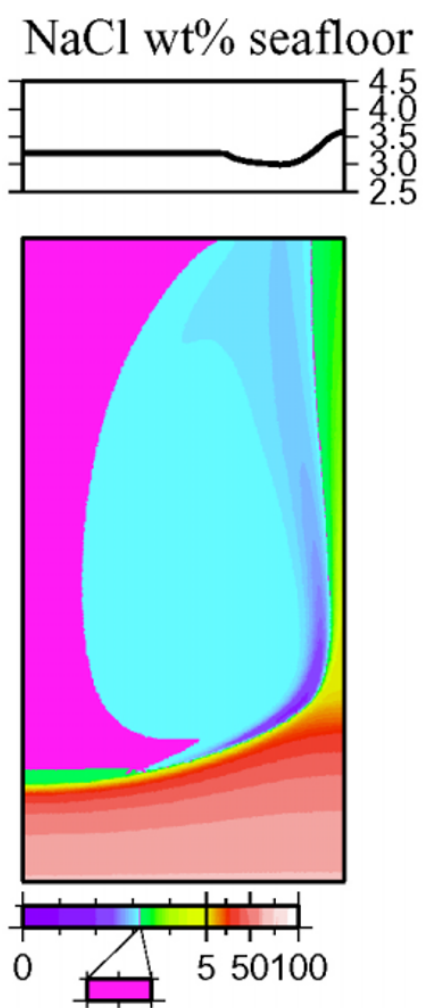

3.193 .21

b) $\mathrm{NaCl} w \mathrm{t} \%$

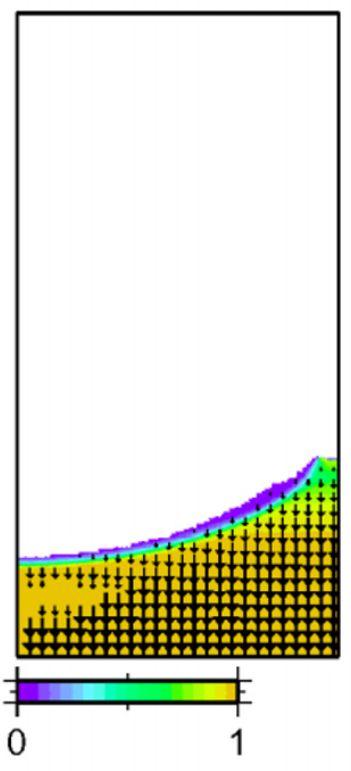

c) Liquid mass fraction $\left(\omega_{1}\right)$

Fig. 4. A typical result of our calculations. The parameters are $R a=100, \Pi=10,1 / L e=1$, and $\phi=0.01$. a) Temperature field. The white thick curves are the streamlines, with the direction of the center-of-mass velocity represented by arrows. The dotted area is the two-phase region. The black arrow at the left side points to the one-phase region in the stagnant layer (see text). Some streamlines pass through the two-phase region. The upper figure shows the temperature of the hydrothermal fluid at the seafloor. b) $\mathrm{NaCl}$ concentration field. To represent the small difference of the concentration, we made the increment of the color palette narrow for the fluids of 0 to $5 \mathrm{wt} \% \mathrm{NaCl}$, and very broad for 5 to $100 \mathrm{wt} \% \mathrm{NaCl}$. The purple color denotes concentrations close to that of seawater, from 3.19 to $3.21 \mathrm{wt} \% \mathrm{NaCl}$. The upper figure shows the $\mathrm{NaCl}$ concentration of the hydrothermal fluid at the seafloor. c) Liquid mass fraction $\left(\omega_{1}\right)$. The black arrows represent the relative velocity between the two-phases. The white area is the one-phase region.

the flow direction. This feature is suitable for our calculations, in which the gradient of $\mathrm{NaCl}$ concentration is approximately perpendicular to the flow direction. The relative advection term in the $\mathrm{NaCl}$ conservation equation (65) is solved by the TVD method with the min-mod function (e.g. Koshizuka, 1997). We use this high-order method because the direction of the relative separation is almost parallel to that of the steepest $\mathrm{NaCl}$ gradient. The upstream direction of this term is judged from the sign of $\left(\omega_{1}-0.5\right)$, because the relative advection term may be rewritten as

$$
\frac{\partial}{\partial z} \frac{\left(C-C_{\mathrm{v}}\right)\left(C-C_{1}\right)}{C_{1}-C_{\mathrm{v}}}\left(\rho_{1}-\rho_{\mathrm{v}}\right)=2\left(\omega_{1}-0.5\right) \frac{\rho_{\mathrm{l}}-\rho_{\mathrm{v}}}{C_{1}-C_{\mathrm{v}}} \frac{\partial C}{\partial z},
$$

if $C_{\mathrm{l}}, C_{\mathrm{v}}, \rho_{\mathrm{l}}$ and $\rho_{\mathrm{v}}$ are constant. Although these quantities are not constant in our calculations, this criterion is good enough for our calculations.

We confirm that the numerical convergence is within $3 \%$ for $\Pi$ between 5 and 20 and for $R a$ of 100 , by changing the number of numerical grid points. The method for evaluating convergence is based on Blankenbach et al. (1989). We carry out numerical calculations for a few choices of numerical grids, and extrapolate the Nusselt number, mean temperature, and the thickness of the stagnant layer, to the limit of infinitesimal mesh width. Our results for $128 \times 256$ mesh is within $3 \%$ of this limit values. The convergence is worse for $\Pi$ out of this range, and its reason is explained at the end of Section 4.

\section{Results}

Figure 4 shows a typical result of the calculations. The parameter values are $R a=100, \Pi=10$, and $1 / L e=1$. A two-layer structure is formed. The upper and lower layers can be defined in terms of streamlines. The boundary between the two layers is the streamline which connects the left and right side boundaries, so that the mean flow described by the center-of-mass velocity $v$ does not cross it. Material is exchanged through the boundary either by diffusion or by the relative motion between the two phases. In the upper layer, circulation is vigorous, and we call it the convection layer. In the lower layer, the flow is almost stagnant, and we call it the stagnant layer. The stagnant lower layer is in contrast with Bischoff and Rosenbauer (1989)'s view of a convective lower brine layer, which is presupposed in several later studies (e.g. Lowell and Germanovich, 1997; Schoofs and Hansen, 2000). It is stagnant because the mean $\mathrm{NaCl}$ concentration $C$ runs almost along the liquidlike branch of the coexisting curve (Fig. 5), and the density of the liquid-like phase $\rho_{1}(z, T)$ increases with temperature along the curve due to the increase of $\mathrm{NaCl}$ concentration. 


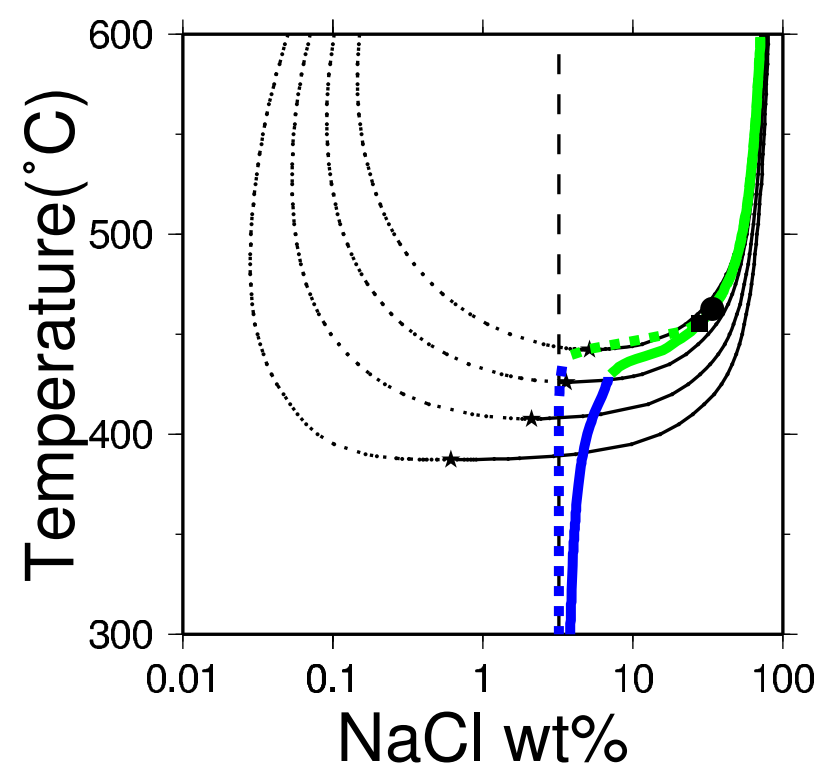

Fig. 5. Temperature and $\mathrm{NaCl}$ concentration along the left downwelling boundary (thick dotted curve) and the right upwelling boundary (thick solid curve) of the calculation area. The vertical axis is the temperature in Celsius degree, and the horizontal axis is the logarithm of $\mathrm{NaCl}$ concentration in wt $\%$. The blue curves signify one-phase fluid, and the green curves signify two-phase fluid. The filled circle and square denote $(C, T)$ at the boundaries between the convection and the stagnant layers. The two-phase co-existing curves for depths of 2500, 3000, 3500 and 4000 $\mathrm{m}$ (corresponding to pressures of 25, 30, 35 and $40 \mathrm{MPa}$, respectively), which are the same as in Fig. 1, are shown.

The brine layer becomes denser downward, and hence stable stratification results.

In the convection layer, seawater flows in from the seafloor, is heated at depth, and returns back up to the sea. The $\mathrm{NaCl}$ concentration of the fluid is almost the same as that of seawater (Fig. 5), because most streamlines do not pass through the two-phase region.

A transition zone is formed near the boundary between the two layers, where the liquid mass fraction has a steep vertical gradient (Fig. 4(c)). The zone consists of both the bottom part of the convection layer and the uppermost part of the stagnant layer. It is where both dilute and dense fluids coexist and separate because of their density difference. The transition zone is thicker in the upwelling area than in the downwelling area. The steep vertical gradient of the salt concentration is established by the balance among the advection due to the mean flow, the downward transport due to the relative velocity, and compositional diffusion. The temperature in the transition zone is about $450^{\circ} \mathrm{C}$. It is close to the boiling or condensation temperature of seawater (Fig. 5), the temperature at which a fluid with seawater salinity crosses the two-phase boundary as temperature rises.

In the stagnant layer, circulation is absent, because a stable density stratification is established. This stagnant layer consists of brine-rich two-phase fluids, except for a small one-phase brine area beneath the downwelling of the upper convection layer (the undotted area to which the black arrow points in Fig. 4). The $\mathrm{NaCl}$ concentrations in the stagnant layer lie close to the liquid-like branch of the two-phase coexisting curve (Fig. 5). This results from the balance of salt transport by the relative velocity between the two phases and

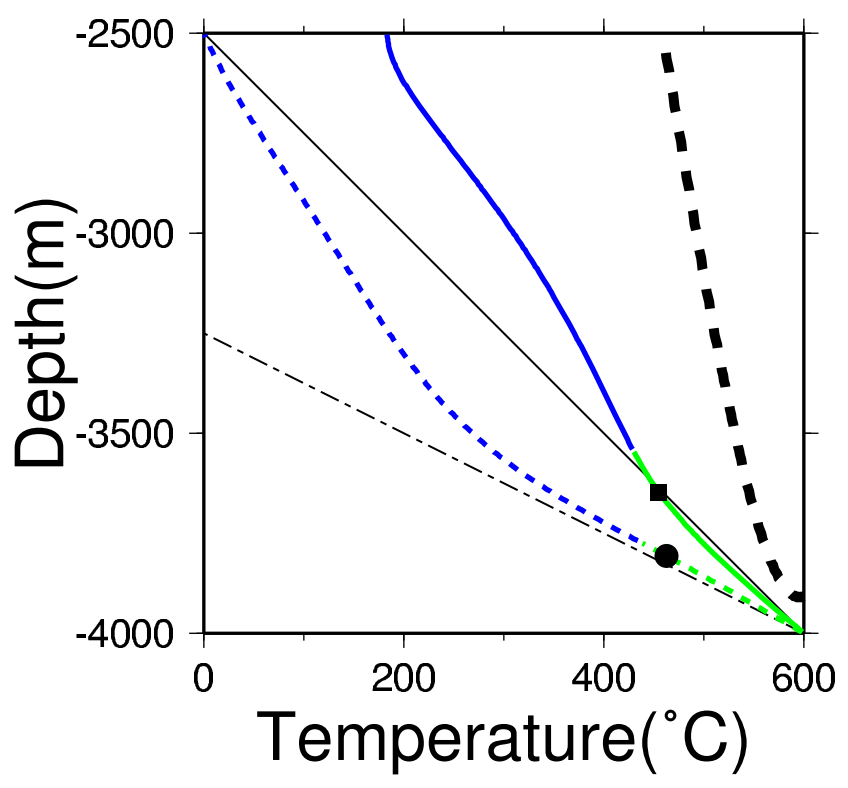

Fig. 6. Temperature as a function of depth along the left downwelling boundary (thick dotted curve) and the right upwelling boundary (thick solid curve) of the calculation area. The parameters for the calculation are $R a=100, \Pi=10$, and $1 / L e=1$. The vertical axis is the depth from the sea surface in meter, and the horizontal axis is the temperature in Celsius degree. The thin solid line shows the conductive temperature profile, and the thin dash-dotted line shows the conductive profile with the thermal conductivity being half. The thick dotted curve represents the vapor-halite-liquid triple point (Palliser and McKibbin, 1998a). Halite precipitates if the temperature of the fluid is higher than this curve. The meaning of the other symbols, colors and patterns of the curves are the same as those in Fig. 5.

compositional diffusion (see Section 5 for details). Since diffusion is weak, salt transport by the relative velocity should be small, and this is accomplished by small vapor fractions, which make this region almost one-phase brine. The average $\mathrm{NaCl}$ concentration is about twenty times that of seawater. Since advection of heat is small in the stagnant layer, the temperature distribution is diffusive, and almost linear with depth (Fig. 6). This layer acts as a thermal insulator and reduces heat transport compared with one-phase convection. Table 1 shows that the Nusselt number $N u$, which is the ratio of the calculated heat flux to the heat flux that the system would give if there were no convection, is reduced to about half of the value for single phase convection $(\Pi=0)$. We shall discuss some implications of this effect for real systems in Section 6.2.

The $\mathrm{NaCl}$ concentration of the hydrothermal fluid at the seafloor is controlled by the structure of the transition zone between the two layers. The competition between the advection by the center-of-mass velocity and the relative motion of the two phases determines the $\mathrm{NaCl}$ concentrations of outgoing fluids. Saline fluids flow out near the ridge axis, because their streamlines pass through deep parts of the transition zone, where the water is more saline than seawater. Dilute fluids discharge more than about $100 \mathrm{~m}$ away from the axis, because their streamlines pass through shallower parts of the transition zone, where downward removal of dense brine makes the water less saline than seawater (Fig. 4). The 

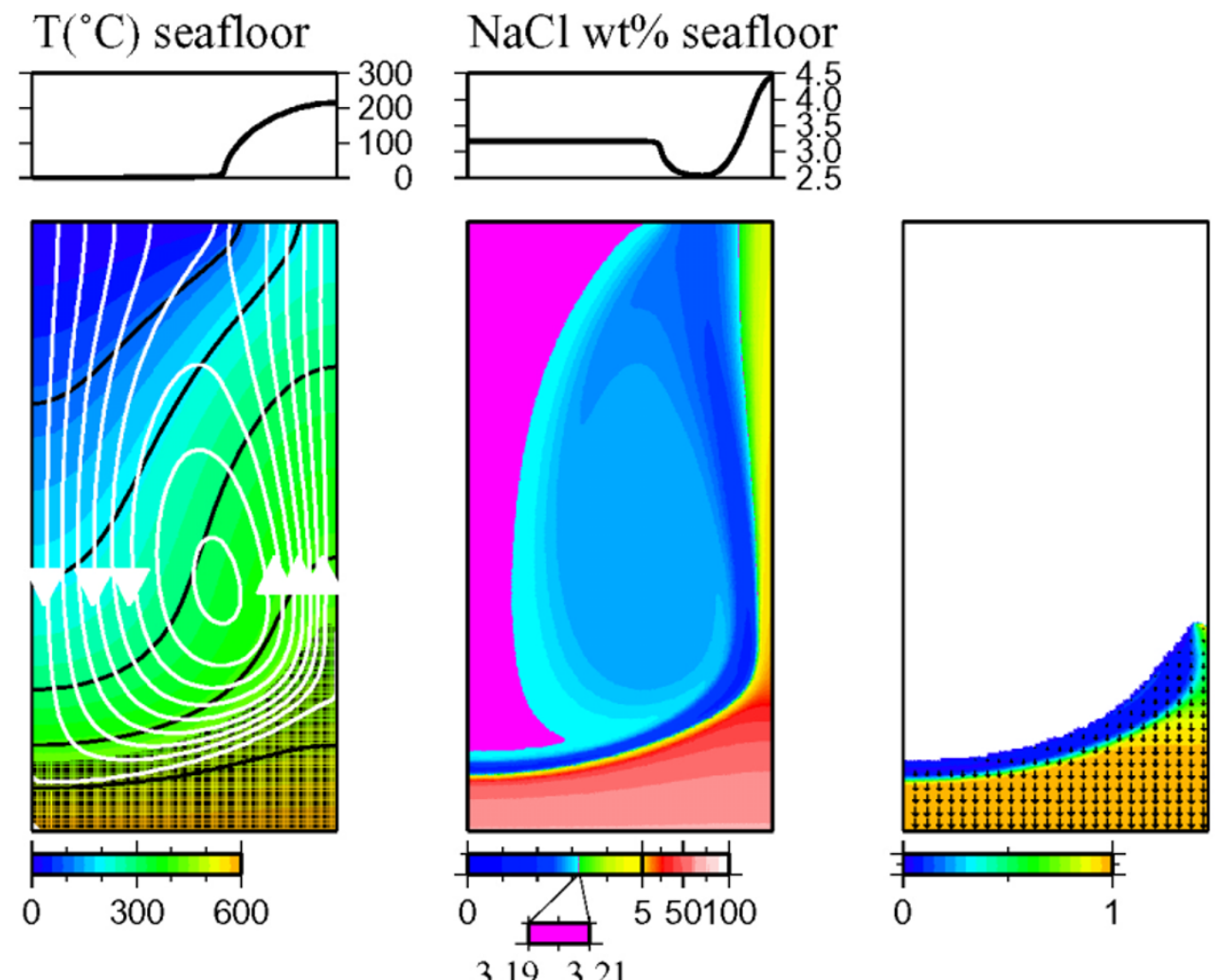

a) Temperature $\left({ }^{\circ} \mathrm{C}\right)$

b) $\mathrm{NaCl} w t \%$

c) Liquid mass fraction $\left(\omega_{1}\right)$

Fig. 7. Same as Fig. 4 but for $\Pi=5$.
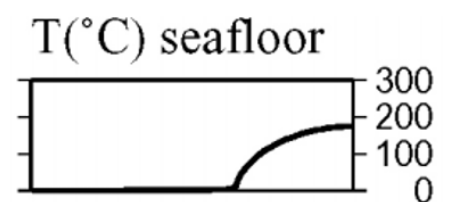

$\mathrm{NaCl}$ wt $\%$ seafloor
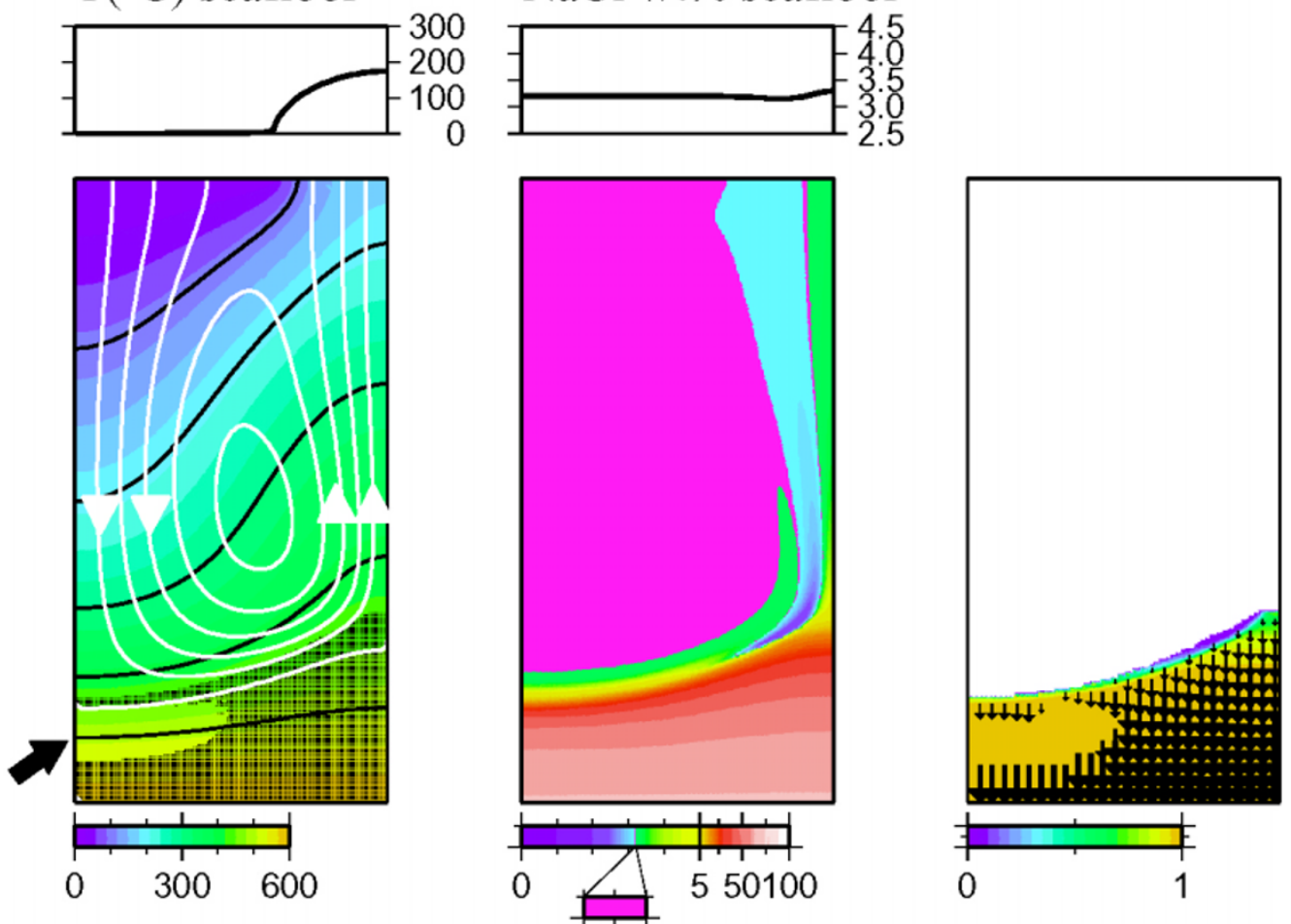
a)Temperature $\left({ }^{\circ} \mathrm{C}\right)$
b) $\mathrm{NaCl} w \mathrm{t} \%$
c) Liquid mass fraction $\left(\omega_{1}\right)$

$\begin{array}{ll}3.19 & 3.21\end{array}$

Fig. 8. Same as Fig. 4 but for $\Pi=20$. 

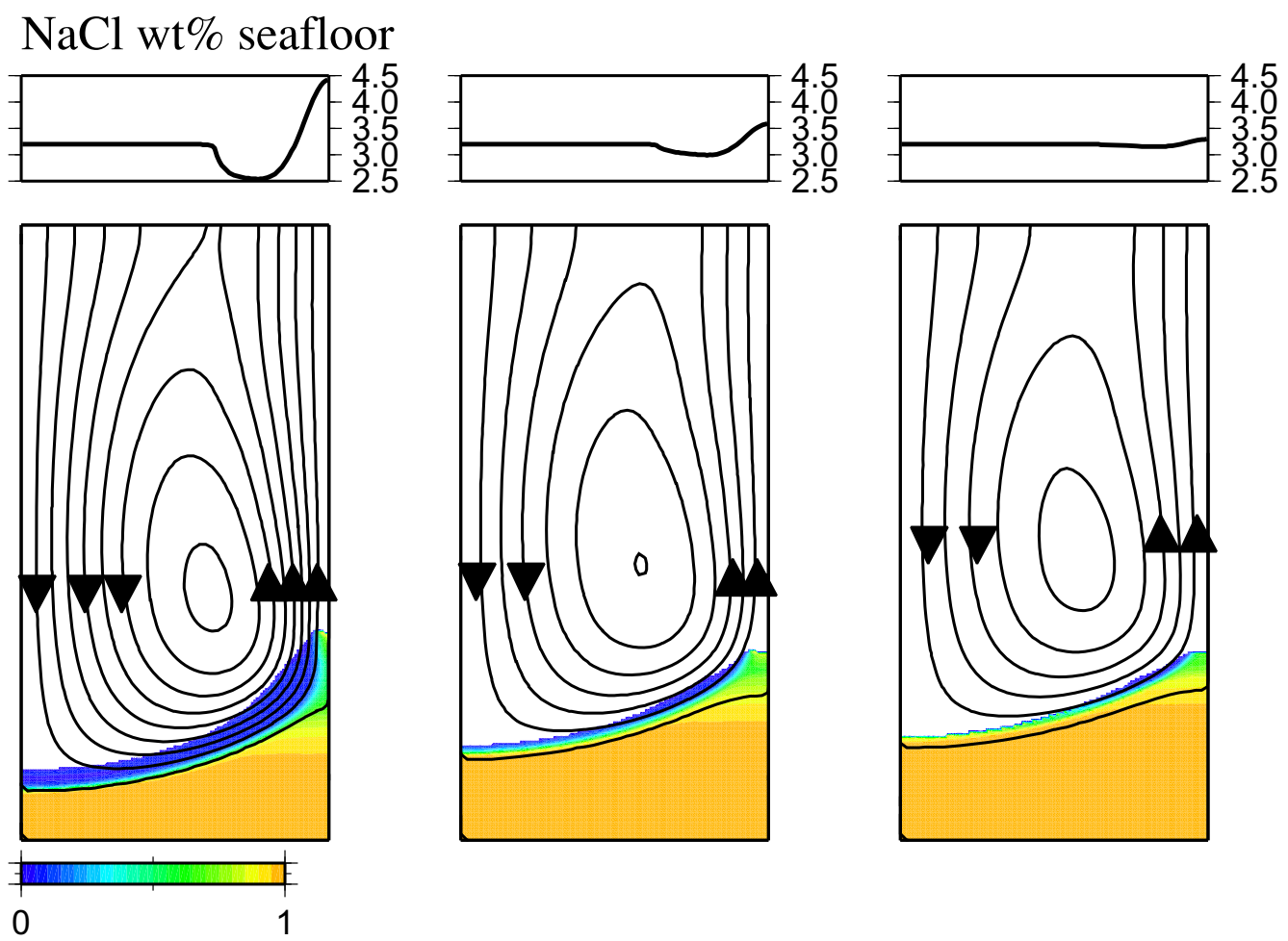

a) $\Pi=5$

b) $\Pi=10$

c) $\Pi=20$

Fig. 9. Comparison of the results for $\Pi=5,10$, and 20. Liquid mass fraction together with streamlines are shown as in Fig. 4. The upper figures show the $\mathrm{NaCl}$ concentrations of the hydrothermal fluid at the seafloor.

weighted horizontally averaged $\mathrm{NaCl}$ concentration

$$
\int_{0}^{A}\left[C\left|v_{z}\right|\right]_{z=1} d x / \int_{0}^{A}\left[\left|v_{z}\right|\right]_{z=1} d x,
$$

in the steady state is the same as that of seawater, if there is no sink or source of the solute in the deep crust, and if compositional diffusion can be neglected at the seafloor, because the total mass of the solute should be conserved.

To further investigate the competition between the relative motion and the mean motion, we perform calculations for two other values of the most relevant parameter $\Pi, 5$ and 20, with $R a$ and $1 / L e$ fixed to the standard values of 100 and 1 , respectively (Table 1, Figs. 7 and 8 ). The parameter $\Pi$ is a measure of the efficiency of phase separation.

Comparison of these results (Fig. 9) shows that the thickness of the transition zone decreases as $\Pi$ increases (phase separation becomes more effective). The thickness can be measured by the separation between the top of the two-phase region and the streamline that connects the left and right side boundaries. $\Pi \sim 10$ marks the transition between two flow regimes: a flow with little phase separation and a flow with almost complete phase separation. This is most notably seen in the range of $\mathrm{NaCl}$ concentration on the seafloor $\Delta C$, because it is controlled by the efficiency of phase separation. For $\Pi=5$, hydrothermal fluids have $\mathrm{NaCl}$ concentrations ranging from 2.5 to $4.4 \mathrm{wt} \% \mathrm{NaCl}$. This difference decreases as $\Pi$ increases (Fig. 10(a)), and almost vanishes when $\Pi=20$, Since the transition zone is thin for large $\Pi$, the number of streamlines that pass through the zone is small, and this gives rise to the decrease in the range of the salinity on the seafloor. In other words, dense brine sinks effectively and cannot rise to the surface.

The reason $\Pi \sim 10$ marks the transition can be understood in terms of the ratio between the magnitude of the relative velocity $\Delta v$ and that of the mean flow velocity $v$ in the transition zone. When this ratio is larger than the order of unity, relative separation occurs effectively, and this leads to complete separation of the convection and stagnant layers. From equations (58) and (61), we have

$$
\frac{\Delta v}{v} \sim \frac{\phi \Delta \rho}{\alpha \rho_{0} \Delta T} \Pi,
$$

where $\alpha$ is the thermal expansion coefficient, $\Delta T$ is the typical temperature difference and $\Delta \rho=\rho_{\mathrm{l}}-\rho_{\mathrm{v}}$ is the typical density difference between the two phases in the transition zone, respectively. The condition $\Delta v / v \geq 1$ is satisfied when $\Pi \geq 12$, since $\Delta T \simeq 450 \mathrm{~K}, \rho_{\mathrm{l}}=780 \mathrm{~kg} \mathrm{~m}^{-3}$, and $\rho_{\mathrm{v}}=250 \mathrm{~kg} \mathrm{~m}^{-3}$ at $3750 \mathrm{~m}$ and $450^{\circ} \mathrm{C}$ (the $(z, C)$ condition of the transition zone), with $\alpha \simeq 10^{-4} \mathrm{~K}^{-1}, \rho_{0}=10^{3} \mathrm{~kg}$ $\mathrm{m}^{-3}$, and $\phi=0.01$. Figure 10 show that the separation of the two layers is almost complete when $\Pi=20$, in accordance with this estimation.

Table 1 and Fig. 10 show the variations of various quantities with $\Pi$. Increasing $\Pi$ results in the increase in the mean thickness of the brine layer $\delta$, and the decreases in the heat flux $N u$, the range of the $\mathrm{NaCl}$ concentration at the seafloor $\Delta C$ (Fig. 10(a), and the maximum temperature at the seafloor $T_{\max }$ (Fig. 10(b). Since the dependence of $\Delta C$ on $\Pi$ is already explained before, we examine the other quantities below. 

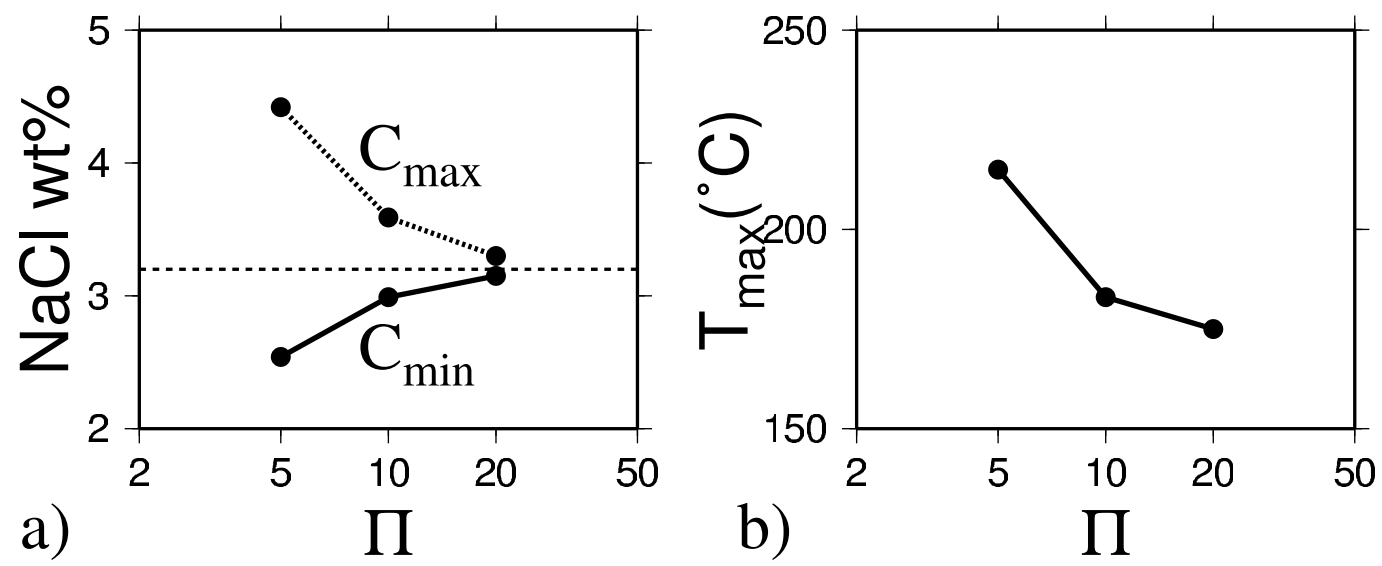

Fig. 10. $\Pi$ dependence of the results. The values of the other parameters are $R a=100,1 / L e=1$ and $\phi=0.01$. a) The maximum and minimum NaCl concentrations of the hydrothermal fluid at the seafloor as a function of $\Pi$. The horizontal dotted line represents the $\mathrm{NaCl}$ concentration of seawater. $\mathrm{b}$ ) The maximum fluid temperature at the seafloor as a function of $\Pi$.

The vigor of convection depends on $\Pi$. Convection becomes vigorous as $\Pi$ decreases. This can be seen in Figs. 7 and 8 or from the Nusselt number $N u$ (Table 1), because a large Nusselt number implies strong convection. Large $\Pi$ results in effective phase separation and effective thermal insulation by the stagnant layer, and hence weakens convection.

The strength of convection is in turn related to the mean thickness of the lower layer $\delta$. The temperature at the top of the stagnant layer is almost the same as the condensation temperature $T_{\mathrm{cs}}$ (Fig. 5). It follows that

$$
\left|\frac{d T}{d z}\right| \simeq \frac{T_{\mathrm{bot}}-T_{\mathrm{cs}}}{\delta}
$$

in the stagnant layer (this equation has a dimension). Hence the Nusselt number, the dimensionless heat flux, which represents the strength of convection, becomes

$$
N u \simeq \frac{T_{\text {bot }}-T_{\mathrm{cs}}}{T_{\text {bot }}-T_{\text {sw }}} \frac{d}{\delta} .
$$

Note that we use $d$ and $T_{\text {bot }}-T_{\mathrm{sw}}$ as the length and temperature scales, respectively. From equation (77), we see that the mean thickness of the layer $\delta$ decreases as $\Pi$ decreases and $N u$ increases.

The maximum temperature at the seafloor $T_{\max }$ depends weakly on $\Pi$ (Fig. 10(b)), because the bottom temperature of the convection layer is almost the same as the condensation temperature of seawater (Fig. 5) irrespective of $\Pi$. The weak dependence can be explained in terms of the strength of convection. When convection is strong, the smearing effect of diffusion is weak, and a high maximum temperature results. To summarize, as $\Pi$ decreases, convection becomes vigorous, and this gives rise to the decrease in $\delta$, and the increases in $N u$ and $T_{\max }$.

Next, we investigate the changes of the results in response to small changes in $R a, \Pi$ and $1 / L e$ in order to obtain a better understanding of the system (Table 3 ). We perturbed the parameters by $\pm 10 \%$ around the reference state for which $R a=100, \Pi=10$, and $1 / L e=1$. Increasing $R a$ results in the decrease in the mean thickness of the brine layer $\delta$, and the increase in the heat flux $N u$, the maximum temperature at the seafloor $T_{\max }$, and the difference of $\mathrm{NaCl}$ concentration at the seafloor $\Delta C=C_{\max }-C_{\min }$. This trend is opposite to increasing $\Pi$. This is because increasing $R a$ makes convection more vigorous and reduces the relative importance of phase separation. Explanations of the effect of changing $\Pi$ are given above. Varying $1 / L e$ does not affect the results much, but increasing $1 / L e$ produces the same trend as in the case of decreasing $\Pi$. The increase in $1 / L e$ means enhanced compositional diffusion, and it reduces the relative importance of phase separation.

We confirm the validity of the assumptions in the calculations, namely, the condition (66) and the neglect of halite precipitation. First, we confirm that the condition (66), which is necessary for equations (58) and (61) to be valid, is satisfied in all the calculations. This condition holds because the phase separation occurs mainly near the critical temperature, where the density difference between the two phases is small. The densities of the liquid and vapor phases are $\rho_{\mathrm{l}}=530 \mathrm{~kg} \mathrm{~m}^{-3}$ and $\rho_{\mathrm{v}}=280 \mathrm{~kg} \mathrm{~m}^{-3}$ at the top of the stagnant layer, whose $(z, T)$ condition is $3750 \mathrm{~m}(37.5 \mathrm{MPa})$ and $450^{\circ} \mathrm{C}$. The liquid mass fraction $\omega_{1}$ is 0.5 around there. Thus, the condition (66) becomes

$$
\Pi \ll \frac{1}{\phi},
$$

since

$$
-\omega_{\mathrm{l}}\left(1-\omega_{\mathrm{l}}\right)\left(\rho_{\mathrm{l}}-\rho_{\mathrm{v}}\right)\left(\frac{1}{\rho_{\mathrm{l}}}-\frac{1}{\rho_{\mathrm{v}}}\right)=0.36<1 .
$$

Our calculations ( $\Pi=5-20$ ) satisfy the condition (78), because the porosity $\phi$ is set at 0.01 .

Second, halite does not precipitate in the steady state in the temperature and pressure (depth) ranges of this study, although we do not explicitly include halite precipitation in our calculations. One type of halite precipitation, in which the temperature of the liquid-vapor two-phase fluid exceeds the liquid-vapor-halite triple point, does not occur, since the fluid does not enter the vapor-halite region (Fig. 6). The other type of halite precipitation, in which the $\mathrm{NaCl}$ concentration of the fluid becomes larger than that of the solubility of $\mathrm{NaCl}$, does not occur, either. This is because $\mathrm{NaCl}$ concentrations 
of the fluid in the stagnant layer lie very close to the liquidlike branch of the co-existing curve, and the concentration on the liquid-like branch is less than that of halite saturation for a given pressure and temperature.

Finally, we explain the dependence of the numerical convergence on the parameter $\Pi$. As we explained in Section 3, the convergence is less than $3 \%$ for $\Pi$ between 5 and 20 , and worsens for larger or smaller $\Pi$. This behavior is due to the structure of the transition zone. The zone becomes thin as $\Pi$ increases. Since the thinnest part of the transition zone contains only five numerical grid points for $\Pi=20$, larger $\Pi$ produces large numerical errors. For $\Pi$ smaller than 5 , the transition zone becomes inclined to the horizontal. Because inclined steep gradient produces large numerical errors for finite difference schemes with Cartesian grids, small $\Pi$ produces large numerical errors. It should however be noted that the qualitative behavior for $\Pi \gg 10$ or $\Pi \ll 10$ can be inferred easily from the results for $\Pi=O(10)$. As $\Pi$ increases from about 10 , the separation of the two layers should become complete. The range of salinities of the vent fluids vanishes for $\Pi \gg 10$ because of effective phase separation (see Fig. 10). As $\Pi$ decreases from about 10, the thickness of the lower layer should decrease. The range of vent-fluid salinities should first increase, then decrease with decreasing $\Pi$, and becomes zero when $\Pi=0$ (one-phase convection; salinity becomes uniform with the seawater value). It thus should have a maximum at some value of $\Pi(<5)$.

\section{Structure of the Stagnant Layer}

We perform vertical one-dimensional steady-state calculations to examine the structure of the lower stagnant layer, which we have found by two-dimensional calculations. Density stratification is stable in the stagnant layer. The fluids are mainly in the two-phase region, and their mean $\mathrm{NaCl}$ concentrations $C$ lie along the liquid-like branch of the two-phase co-existing curve. We examine how this salinity distribution is formed in this section. One-dimensional analysis is particularly useful for this region, because the advection by the center-of-mass velocity $v$ is small. Bai et al. (2003) qualitatively discussed the balance of $\mathrm{NaCl}$ transfer in the bottom brine layer for their one-dimensional pipe model. Our analysis here extends their discussion quantitatively.

The steady-state one-dimensional basic equations are obtained from the two-dimensional equations (57), (58), (59) and (65) by letting $v=0$ because the lower layer is stagnant. Accordingly, the same approximations as those for the twodimensional systems are used. The equations are written as

$$
\frac{d^{2} T}{d z^{2}}=0
$$

and

$$
-B \frac{d}{d z}\left[\omega_{\mathrm{l}}\left(1-\omega_{1}\right)\left(C_{1}-C_{\mathrm{v}}\right)\left(\rho_{1}-\rho_{\mathrm{v}}\right)\right]=\frac{d^{2} C}{d z^{2}},
$$

where $B$ is defined as

$$
B=\Pi R a L e=\frac{\rho_{0} g k d}{\mu_{0} D} .
$$

The parameter $B$ is the ratio of the timescale of compositional diffusion $d^{2} / D$ to that of the relative separation be- tween the two phases $d \mu_{0} / \rho_{0} g k$, and is the only one parameter in the one-dimensional system. A large $B$ results in effective relative separation of the two phases. Here, $\rho_{\mathrm{l}}, \rho_{\mathrm{v}}, C_{\mathrm{l}}$, and $C_{\mathrm{v}}$ in equation (81) are expressed as functions of temperature and depth as we explained in Section 2.1.6. Note that the liquid-phase velocity $v_{\mathrm{l}, z}(z)$ and the vapor-phase velocity $v_{\mathrm{v}, z}(z)$ are non-zero (see equation (4), the definition of the center-of-mass velocity), although the center-of-mass velocity $v_{z}(z)$ is zero. We solve these equations in the twophase stagnant layer and the one-phase region just above the stagnant layer where the center-of-mass velocity $\boldsymbol{v}$ is small.

Equation (80) implies that the temperature field has a constant gradient. Heat is transfered only by conduction and advective heat transport is negligible. From equation (80), we have

$$
T(z)=T_{\text {bot }}-F z,
$$

where $T_{\text {bot }}$ is the non-dimensional temperature at the brittleductile front $(z=0)$, and $F$ is the non-dimensionalized temperature gradient. Here $F=1$ signifies the conductive gradient when the flow is absent in the whole region. We assume that $F=1.5$ in the following one-dimensional calculations. This value is taken from the result of the two-dimensional calculation for $R a=100,1 / L e=1$ and $\Pi=10$ (Fig. 6).

We integrate equation (81) to obtain

$$
\frac{d C}{d z}=-B \omega_{1}\left(1-\omega_{1}\right)\left(C_{1}-C_{\mathrm{v}}\right)\left(\rho_{1}-\rho_{\mathrm{v}}\right)+\text { const. }
$$

which means that the $\mathrm{NaCl}$ concentration is maintained by the balance between downward salt transport and upward salt diffusion, as Bai et al. (2003) pointed out. We use the boundary condition (55) at $z=0$ to find that the constant in equation (84) is 0 . The upper boundary condition we adopt for solving equation (84) is

$$
C=C_{\mathrm{sw}} \text { at } z=1 .
$$

This means that the $\mathrm{NaCl}$ concentration in the one-phase region far above the stagnant layer is the same as that of seawater. This results from equation (84) because the right hand side is zero in a one-phase region. This boundary condition (85) is justified by effective convective mixing in the upper layer, where the salinity is almost uniform at the seawater value in our two-dimensional calculations (e.g. Fig. 4).

We numerically integrate equation (84) from the seafloor $z=1$ to the base $z=0$ for $B$ of $0,5,10,20,50,100$, and 1000 , to obtain one-dimensional profiles of the $\mathrm{NaCl}$ concentration of the fluid. The results are shown in Fig. 11. The $\mathrm{NaCl}$ concentration is shown as functions of depth and temperature. Since the temperature gradient is constant, the spatial gradient of the $\mathrm{NaCl}$ concentration (Fig. 11(a)) and the gradient of the temperature-concentration curve (Fig. 11(b)) are proportional, namely,

$$
\frac{d T}{d C}=-F\left(\frac{d C}{d z}\right)^{-1}
$$

The thickness of the stagnant layer is about $250 \mathrm{~m}$, which is easily explained by using equation (83). The thickness is approximately given by the height at which the temperature is the condensation temperature of seawater $T_{\mathrm{cs}}$, as

$$
\delta \simeq \frac{T_{\text {bot }}-T_{\mathrm{cs}}}{F} .
$$



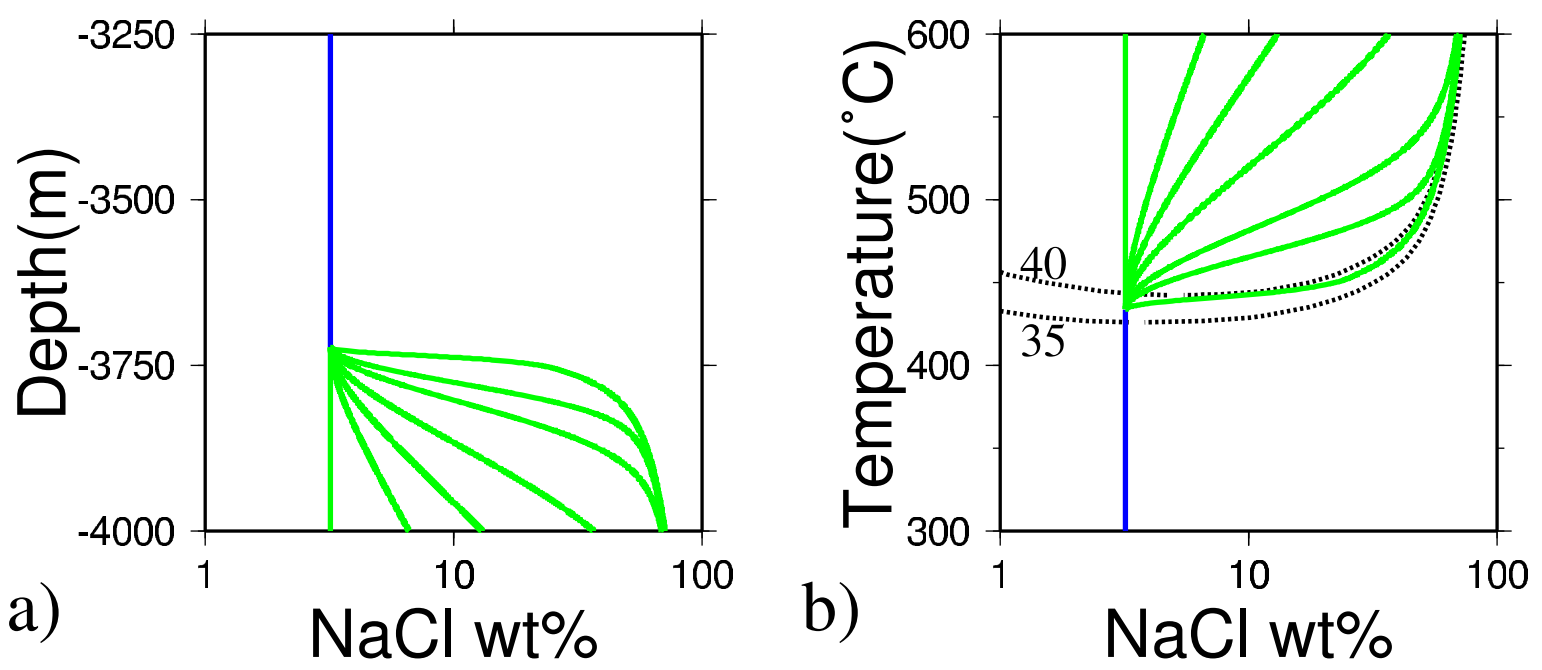

Fig. 11. Results of one-dimensional calculations for $B=0,5,10,20,50,100$, and 1000, from left to right in the figure. The non-dimensionalized temperature gradient $F$ for the calculation is 1.5. a) depth from the seafloor against $\mathrm{NaCl}$ concentration in wt\%. The latter is in a logarithmic scale. b) temperature in Celsius degree against $\mathrm{NaCl}$ concentration in wt $\%$. The latter is in a logarithmic scale. The meaning of the other symbols, patterns and colors of the curves are the same as those in Fig. 5. Note that $B=0$ corresponds to one-phase thermal conduction.

It becomes about $(4 F)^{-1}$, because the condensation temperature of seawater $T_{\mathrm{cs}}$ is about $450^{\circ} \mathrm{C}$ in a dimensional value and $3 / 4$ in a non-dimensional value, and the bottom temperature $T_{\text {bot }}$ is $600^{\circ} \mathrm{C}$ in a dimensional value and 1 in a nondimensional value. When $F=1.5$, the thickness $\delta$ is about 0.17 , which is about $250 \mathrm{~m}$ in a dimensional value.

Profiles of the $\mathrm{NaCl}$ concentration for $B \gg 1$ may be explained as follows. The $\mathrm{NaCl}$ concentration of the fluid is the same as that of seawater in the one-phase region and at the top of the two-phase region, where $\omega_{1}=0$, and increases with depth in the two-phase region $(d C / d z<0)$, because the right hand side of equation (84) is always negative. The change in the gradient of $\mathrm{NaCl}$ concentration is explained by the right hand side of equation (84) being proportional to $\omega_{1}\left(1-\omega_{1}\right)$. For a given pressure and temperature (depth), the gradient of $\mathrm{NaCl}$ concentration of the fluid is maximum at $\omega_{1}=0.5$, and decreases as the $\mathrm{NaCl}$ concentration of the fluid approaches the two-phase co-existing curve, $\omega_{1}=1$ or $\omega_{1}=0$. Therefore, from the top of the two-phase region, the absolute value of the $\mathrm{NaCl}$ gradient first increases with depth, becomes maximum near a depth where $\omega_{1}=0.5$, and then decreases with depth. At the bottom, the $\mathrm{NaCl}$ concentration of the fluid comes close to the liquid-like branch of the twophase co-existing curve, $\omega_{1}=0$.

The density stratification is stable in the two-phase region. This is because the $\mathrm{NaCl}$ concentrations of the fluid in this layer lie close to the liquid-like branch of the two-phase coexisting curve, and the density along this curve increases with temperature (Fig. 2).

We now examine the $B$-dependence of the results. In the one-dimensional calculations, the $\mathrm{NaCl}$ concentration curve approaches the liquid-like branch of the two-phase coexisting curve, as $B$ increases (Fig. 11(b)). This can be understood by examining the basic equation (84). We use equation (44) to rewrite (84) as

$$
\frac{d C}{d z}=-B \cdot\left(\rho_{1}-\rho_{\mathrm{v}}\right) \frac{\left(C-C_{\mathrm{v}}\right)\left(C_{1}-C\right)}{C_{1}-C_{\mathrm{v}}} .
$$

If $C_{1}, C_{\mathrm{v}}, \rho_{\mathrm{l}}$, and $\rho_{\mathrm{v}}$ are constants, equation (88) has an analytical solution

$$
C=C_{1}-\frac{C_{1}-C_{\mathrm{v}}}{1+\gamma \exp \left[-B\left(\rho_{1}-\rho_{\mathrm{v}}\right) z\right]},
$$

where $\gamma$ is a constant. This solution shows that the $\mathrm{NaCl}$ concentration of the fluid approaches the liquid-like branch of the two-phase co-existing curve $\left(C \rightarrow C_{1}\right)$ with depth (as $z$ decreases), and that the rate of the approach increases with $B$ and $\left(\rho_{\mathrm{l}}-\rho_{\mathrm{v}}\right)$.

We compare the two-dimensional calculation for $R a=$ $100,1 / L e=1$ and $\Pi=10$ (Figs. 4 and 5), with the onedimensional result for $B=1000$ (Fig. 12). Note that the comparison is meaningful only in the stagnant layer, where the two-dimensional center-of-mass velocity is negligible. In Fig. 12, the plots of the two-dimensional result are the profiles of temperature, $\mathrm{NaCl}$ concentration, and density along the central line, $x=A / 2$. We find that the plots of onedimensional and two-dimensional results (Fig. 12(a)-(c)) agree well in the stagnant layer. The agreement of the temperature profiles (Fig. 12(d)) is a matter of course, because the temperature gradient $F=1.5$ in the one-dimensional calculation is chosen so that it should agree with that in the stagnant layer in the two-dimensional calculation. The agreement of the $\mathrm{NaCl}$ concentration profiles confirms that upward compositional diffusion and the downward salt transport by the relative separation are almost balanced.

\section{Discussion}

\subsection{Temperature of the vent fluid}

The stability of the maximum temperatures of black smokers at around $350^{\circ} \mathrm{C}$ (e.g. Wilcock, 1998) is one of the reasons that Bischoff and Rosenbauer (1989) proposed the model of two-layer circulation. If phase separation occurs, the vent temperature is determined by the phase separation temperature. Many other mechanisms have been proposed to explain the temperature: (1) brittle-ductile transition of the oceanic crust (Fournier, 1987), (2) silica and 

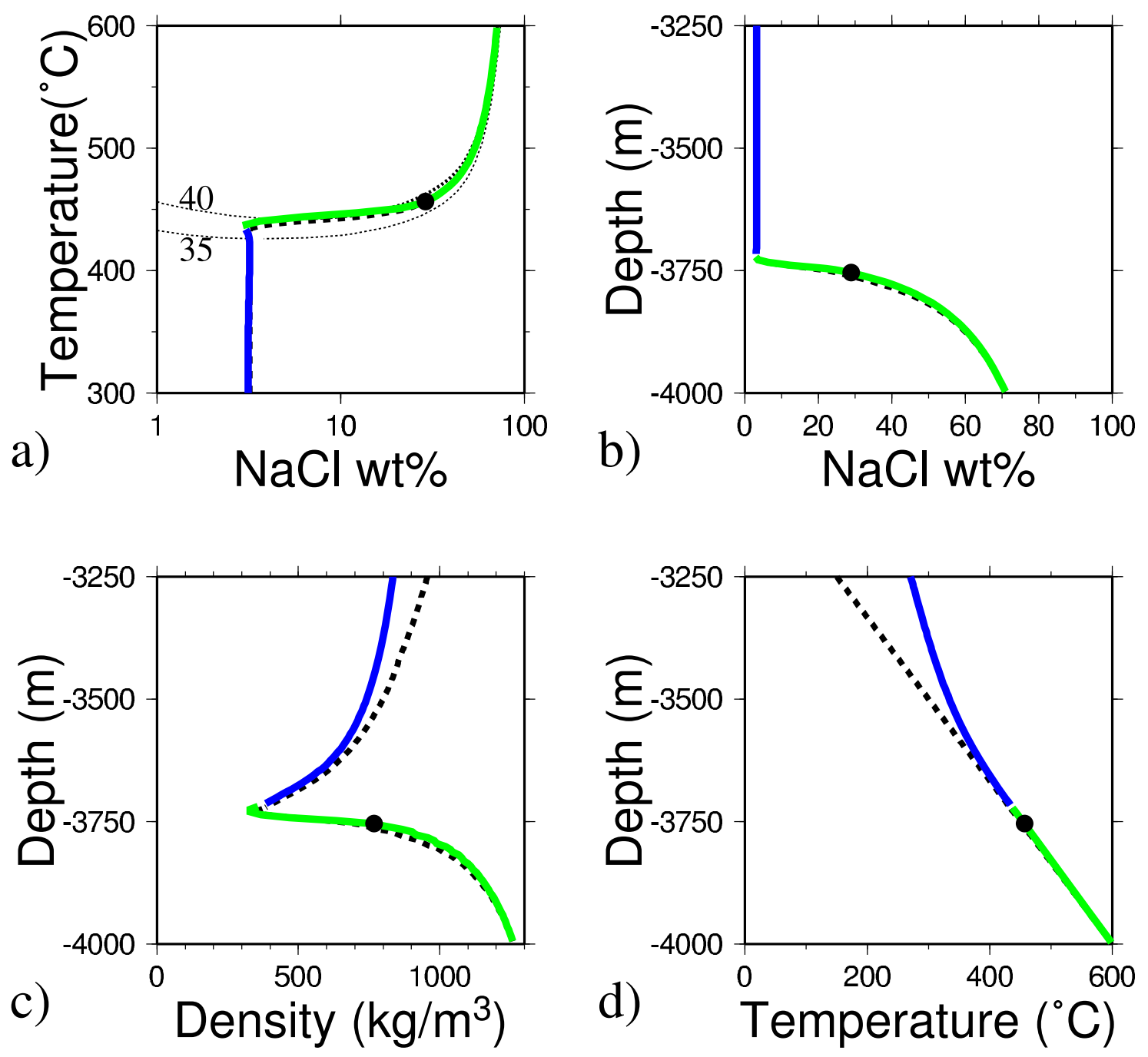

Fig. 12. Comparison of the results of one-dimensional and two dimensional calculations. a) Temperature against $\mathrm{NaCl}$ concentration, b) depth against $\mathrm{NaCl}$ concentration, c) depth against density, and d) depth against temperature. The parameters used in the calculations are $R a=100, \Pi=10$, and $1 / L e=1(B=1000)$. The thin black dotted curves in a) signify the two-phase co-existing curves, which are the same as those in Fig. 5. The thick dotted curves are the result of the one-dimensional calculation for a non-dimensionalized temperature gradient $F$ of 1.5 . The colored solid curves signify the two-dimensional result along $x=0.25$ (the center of the calculation area). The one- and two-dimensional results are almost identical in the two-phase region and in the one-phase region just above the two-phase region. The meanings of the other colors and symbols are the same as those in Fig. 5.

anhydrite precipitation in the pore space (Martin and Lowell, 2000; Fontaine et al., 2001), (3) large changes of physical properties of water around the critical point (Wilcock, 1998; Jupp and Schultz, 2000), and (4) the development of a two-layer structure by phase separation (Bischoff and Rosenbauer, 1989). Among the four mechanisms, (2)-(4) are related to the properties of water around the critical point. Therefore, these effects can not be separated. In our model, the effects of (4) and part of (3) are included.

From our calculations, we confirm that the temperature at the bottom of the upper layer becomes close to the condensation temperature of seawater, $450^{\circ} \mathrm{C}$ (see Fig. 5). This is because efficient phase separation and vigorous convection make the upper layer compositionally uniform at the $\mathrm{NaCl}$ concentration of seawater. This result vindicates the importance of the mechanism (4). The vent temperature consequently becomes the condensation temperature of seawater at most. For the calculations of this study ( $R a=100$, $\Pi=5-20,1 / L e=1)$, the maximum venting temperature is around $200^{\circ} \mathrm{C}$. The temperature drop from the phase separation temperature is due to thermal diffusion. As shown in Table 3, the maximum temperature depends most notably on the Rayleigh number $R a$, which is the ratio of the timescale of the heat advection by the center-of-mass velocity $v$ to that of thermal diffusion. This shows the importance of thermal diffusion on the temperature distribution. Higher Rayleigh number would lead to higher vent temperature.

The effect of (3) is to concentrate the upflow and to produce higher vent temperature. The critical properties of water change markedly around the critical point of water: the thermal conductivity drops sharply with increasing temperature, the kinematic viscosity exhibits a minimum, and the heat capacity shows a maximum near the critical point (Haar et al., 1984). Wilcock (1998) showed that all these properties tend to decrease the temperature difference between the bottom of the circulation layer and the vent fluid. He demonstrated that the vent temperature is about $0.5-0.65$ times the bottom temperature when these properties are included, 
Table 3. Changes of the results in response to small changes ${ }^{\mathrm{a}}$ of $R a, \Pi$, and $1 / L e$.

\begin{tabular}{c|cccc}
\hline & $N u$ & $\Delta C$ & $T_{\max }-T_{\mathrm{sw}}$ & $\delta$ \\
\hline$R a \times 1.1$ & $\times 1.14$ & $\times 1.07$ & $\times 1.08$ & $\times 0.875$ \\
$R a \times 0.9$ & $\times 0.866$ & $\times 0.777$ & $\times 0.901$ & $\times 1.16$ \\
\hline$\Pi \times 1.1$ & $\times 0.988$ & $\times 0.870$ & $\times 0.991$ & $\times 1.02$ \\
$\Pi \times 0.9$ & $\times 1.02$ & $\times 1.16$ & $\times 1.01$ & $\times 0.981$ \\
\hline $1 / L e \times 1.1$ & $\times 1.01$ & $\times 1.00$ & $\times 1.01$ & $\times 0.990$ \\
$1 / L e \times 0.9$ & $\times 0.992$ & $\times 1.00$ & $\times 0.994$ & $\times 1.01$ \\
\hline
\end{tabular}

${ }^{a}$ The solution used as a standard is for the parameters of $R a=100, \quad \Pi=10$, and $1 / L e=1$. The magnitudes of the perturbations for $R a, \Pi$, and $1 /$ Le are $\pm 10 \%$.

whereas it is less than 0.5 times the bottom temperature when these effects are absent. If we apply this relationship to our calculations with the bottom temperature of $430^{\circ} \mathrm{C}$ and the multiplication factor of 0.65 , the maximum vent temperature becomes $280^{\circ} \mathrm{C}\left(=430^{\circ} \mathrm{C} \times 0.65\right)$, about $80^{\circ} \mathrm{C}$ higher than our results.

\subsection{Heat efficiency}

Here we first show that hydrothermal heat transport at mid-ocean ridges is lower than what is expected for one phase convection. We then argue that this suppression is due to two-layer structure found in our study, but quantitatively this effect may not be enough to explain observations.

First we compare heat transport for one-phase convection and the heat flow at mid-ocean ridges, and show that the latter is considerably lower than the former. For onephase porous media with isothermal top and bottom boundaries, the relationship between the Nusselt number and the Rayleigh number is given experimentally by Elder (1967) as

$$
N u=R a_{n} / 40,
$$

where $R a_{n}$ is the conventional Rayleigh number defined in Section 2.3. Cherkaoui and Wilcock (1999) recently obtained another relationship from their numerical simulations of thermal convection in an open-top porous layer heated from below as

$$
N u \propto R a_{n}^{0.67-1.29},
$$

where the power depends on the mode of convection. We use the former relationship (90), because the Rayleigh numbers used in the latter study were not high enough to ensure an asymptotic relation. Equation (90) yields the estimate of the Nusselt number in the range from $10^{4.5}$ to $10^{7.5}$ for the range of the Rayleigh number around blacksmoker fields which is probably between $10^{6}-10^{9}$ as we explain below. On the other hand, the Nusselt number $N u$ estimated from the observations of heat flux around the ridge axis ranges from 10 to $10^{3}$ as we explain below. Therefore, the heat flux at mid-ocean ridges is suppressed compared with one-phase thermal convection.

The Nusselt number is estimated from the observed heat flux as follows. The heat flow of a hydrothermal vent field is about 10-10 $30^{3}$ MW (Lowell et al., 1995), and the area of a vent field is about $10^{6} \mathrm{~m}^{2}$ (Lowell et al., 1995). This gives the heat flow density of about $10-10^{3} \mathrm{Wm}^{-2}$. The conductive heat flux is estimated as $1 \mathrm{Wm}^{-2}$ from the expression $\lambda \Delta T / \Delta z$, where we use the heat conductivity of the rock $\lambda=2.5 \mathrm{Wm}^{-1} \mathrm{~K}^{-1}$, the temperature difference $\Delta T=600$
$\mathrm{K}$, and the height of circulation $\Delta z=1500 \mathrm{~m}$. These values give the estimate of the Nusselt number to be $10-10^{3}$.

Estimates of the Rayleigh number from observed quantities have large uncertainties. In Section 3 (Table 2), we estimated that the Rayleigh number is between $10^{\circ}$ and $10^{9}$, because permeabilities estimated from observations range from $10^{-8} \mathrm{~m}^{2}$ to $10^{-17} \mathrm{~m}^{2}$ (Nehlig and Juteau, 1988; Becker, 1989). The low permeabilities of $10^{-17}-10^{-14} \mathrm{~m}^{2}$ come from the off-axis drilled core from the Hole 504B (Becker, 1989). We argue that these values are too small for the onaxis permeability because off-axis fractures would already have been filled with secondary minerals. On the other hand, the high permeabilities of $10^{-11}-10^{-8} \mathrm{~m}^{2}$ come from the observation of the Samail ophiolite (Nehlig and Juteau, 1988; Nehlig, 1994), which would represent on-axis remains of hydrothermal circulation. Thus we believe that the Rayleigh number of $10^{6}-10^{9}$ is appropriate for on-axis circulation.

Next we consider the mechanism of this heat flow suppression. A stagnant brine layer found in this study is one of the candidate, but many other mechanisms can lower the heat flow. For example, (1) two-layer convection (Bischoff and Rosenbauer, 1989), (2) double-diffusive convection of correlated temperature-salinity fluids (e.g. Schoofs et al., 2000), and (3) precipitation of anhydrite and/or silica minerals in the pore space.

Here we discuss implications of our results in terms of heat efficiency. We have shown that the heat flux is suppressed for two-layer convection. However, the difference in heat flux between one-layer and two-layer convection in our calculations is much smaller that that estimated from observations and from equation (90). The latter is three or four orders of magnitude, while the former is only a factor of two for the parameters we used (compare the Nusselt numbers for $\Pi=0$ and $\Pi=10$ in Table 1). Calculations with wider ranges of the parameters $R a, \Pi$, and $1 / L e$, would be necessary to clarify how much heat transport is suppressed by phase separation.

\subsection{Spatial variation of the salinity in a vent field}

From our calculations, fluids with different salinities are expected in one vent field (Fig. 10). Fluids which vent near the ridge axis will have salinities higher than those of seawater, whereas fluids which vent more than about $100 \mathrm{~m}$ away from the axis will have salinities lower than those of seawater. In a steady state, the mean salinity of vent fluids should be the same as that of seawater, and high salinity fluids should be balanced by low salinity fluids. However, observed salinities of a vent field are either higher or lower 
than that of seawater in most cases (e.g. Von Damm, 1995). This would be because most fluid samples are collected at strong upflow regions.

Exceptions were found at ASHES vent field (Butterfield et al., 1990), and at EPR $21^{\circ} \mathrm{S}$, (Von Damm et al., 2003), where salinities both higher and lower than that of seawater were observed in one vent field. Butterfield et al. (1990) interpreted this phenomenon as due to phase separation in a crack along the caldera wall. On the other hand, Von Damm et al. (2003) interpreted that phase separation occurs within the sulfide structure, since no water-rock interaction after the phase separation is evidenced by their chemical analysis of major elements in the fluids. These interpretations are similar to our results in that the salinity variation is caused by phase separation in circulation, and does not require temporal variation.

It is to be noted again that there should be a counterpart for fluids whose salinities are different from that of seawater. The counterpart can emerge as temporal changes or spatial changes as in our calculations, or may not be observed if the counterpart is hidden deep in the crust. Without observations over broad areas including off-axis fluids, it cannot be concluded which is the case. However, the stability of compositions of many vent fluids (e.g. Von Damm, 1995) implies that many vent fields are in a steady state. This, with our results, suggests that there should be discharge with counterpart compositions somewhere off-axis.

6.4 Effects of the latent heat of condensation and the heat capacity difference between the two-phases

First we discuss the effect of the latent heat of condensation on the steady-state temperature structure of the twophase region. The non-dimensional latent heat of condensation, which is the second term of the right hand side of the energy conservation equation (35), is written as

$$
\begin{aligned}
& R a|L| \phi \boldsymbol{\nabla} \cdot\left(\omega_{1} \boldsymbol{v}_{\mathrm{l}}\right) \\
& \quad=R a|L| \phi\left\{\boldsymbol{v} \cdot \boldsymbol{\nabla} \omega_{1}-\Pi \frac{\partial}{\partial z}\left[\omega_{1}\left(1-\omega_{1}\right)\left(\rho_{1}-\rho_{\mathrm{v}}\right)\right]\right\},
\end{aligned}
$$

using the Boussinesq approximation. Here, $L=$ $\Delta h /\left(c_{p} \Delta T\right) \leq 0$ is the non-dimensionalized latent heat of condensation. In the temperature and pressure (depth) range of this study, $|L|$ is at most 0.3 , since $c_{p}=5 \times 10^{3} \mathrm{~J} \mathrm{~kg}^{-1}$ $\mathrm{K}^{-1},|\Delta h| \leq 2 \times 10^{6} \mathrm{~J} \mathrm{~kg}^{-1} \mathrm{~K}^{-1}$ (Palliser and McKibbin, $1998 \mathrm{~b}$ ), and $\Delta T=600 \mathrm{~K}$. Equation (92) shows that the latent heat affects the energy balance in the region where the liquid mass fraction $\omega_{1}$ varies.

We argue that latent heat would be a secondary effect in the steady state. In our calculations, the spatial variation of the liquid mass fraction $\omega_{1}$ is large in the thin transition zone above the stagnant layer (e.g. Fig. 4). Since both condensation (exothermic) and vaporization (endothermic) processes occur within this thin zone, the effect of latent heat on the total heat budget should be small. Moreover, $(C, T)$ conditions in the transition zone are close to the critical point of the $\mathrm{NaCl}-\mathrm{H}_{2} \mathrm{O}$ system (Fig. 5), around which the latent heat $\Delta h$ is small (Palliser and McKibbin, 1998b). This also makes the contribution of latent heat small.

Next we discuss the effect of the heat capacity difference between the liquid and vapor phases. The difference may be important because the relative motion of the two phases transports heat when the two heat capacities are different. The effect is expressed by the last term of the left-hand side of the equation (35). The term is rewritten in a nondimensionalized form as

$$
\begin{aligned}
& \phi R a\left(c_{p, 1}-c_{p, \mathrm{v}}\right) \omega_{\mathrm{l}}\left(1-\omega_{\mathrm{l}}\right)\left(\boldsymbol{v}_{\mathrm{l}}-\boldsymbol{v}_{\mathrm{v}}\right) \cdot \nabla T \\
& \quad=-\phi R a \Pi\left(c_{p, 1}-c_{p, \mathrm{v}}\right) \omega_{\mathrm{l}}\left(1-\omega_{\mathrm{l}}\right)\left(\rho_{\mathrm{l}}-\rho_{\mathrm{v}}\right) \frac{d T}{d z} .
\end{aligned}
$$

Here $\left(c_{p, 1}-c_{p, \mathrm{v}}\right)$ is the non-dimensionalized heat capacity difference, which is usually negative. We estimate that $\left(c_{p, 1}-c_{p, \mathrm{v}}\right) \simeq-3$ by taking the maximum and the minimum heat capacity of pure water (Haar et al., 1984) at typical pressure and temperature conditions around the transition zone ( $35 \mathrm{MPa}$ and $450^{\circ} \mathrm{C}$ ), if it is non-dimensionalized by the heat capacity at ambient temperatures $\left(4 \mathrm{~kJ} \mathrm{~K}^{-1} \mathrm{~kg}^{-1}\right)$.

We argue that the effect of the heat capacity difference between the two phases on the temperature field is small. The one-dimensional energy equation becomes

$$
-\phi R a \Pi\left(c_{p, 1}-c_{p, \mathrm{v}}\right) \omega_{1}\left(1-\omega_{1}\right)\left(\rho_{1}-\rho_{\mathrm{v}}\right) \frac{d T}{d z}=\frac{d^{2} T}{d z^{2}},
$$

in a non-dimensional form if we neglect the advection by the mean flow. Assuming that the coefficient of $d T / d z$ is a constant, this equation is integrated as

$$
\frac{d T}{d z}=-\phi R a \Pi\left(c_{p, 1}-c_{p, \mathrm{v}}\right) \omega_{1}\left(1-\omega_{1}\right)\left(\rho_{1}-\rho_{\mathrm{v}}\right) T-F
$$

where $F$ is the temperature gradient without the heat transport due to the relative motion, and is about 1.5 as we explained in Section 5. The coefficient of $T$ in the first term of the right-hand side is 4 at most, for $\phi=0.01$, $R a=100$, and $\Pi=20$, which are used in the calculation, and $\rho_{\mathrm{l}}-\rho_{\mathrm{v}}=0.25$ and $\omega_{\mathrm{l}}=0.5$, which are typical values around the transition zone. The temperature $T$ is unity at most, and thus the effect of the heat transport due to the heat capacity difference on the temperature gradient can be large, and may lead to positive temperature gradient. However, this does not affect the temperature much, because the transition zone, where relative motion exists, is narrow with the width of about 0.05 in our calculations.

\section{Conclusion}

We numerically investigate mid-ocean ridge hydrothermal circulation, with emphasis on dynamical effects of the phase separation of seawater. The phase separation of seawater is found to be crucial in determining the temperature and $\mathrm{NaCl}$ concentration of the vent fluid, and the heat efficiency of circulation. The phase separation results in a two-layer structure with an upper convection layer and a lower saline stagnant layer. The key quantity that governs the structure is the ratio of the magnitude of the relative velocity between the liquid and vapor phases to that of the mean flow velocity. When this ratio is less than unity, the development of the brine layer is incomplete. When it is more than unity, the separation of the two layers is almost complete. Vent fluids both more and less saline than seawater are formed. They are more saline near the ridge axis, and less saline in the area more than about $100 \mathrm{~m}$ away from the axis. The 


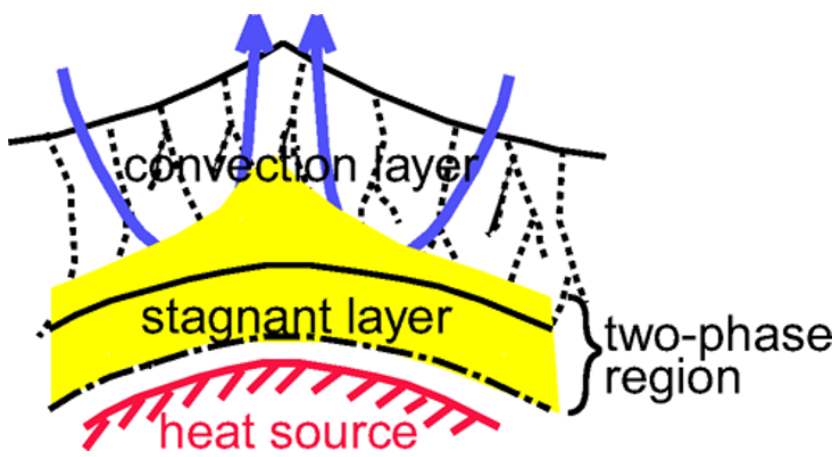

Fig. 13. Schematic picture of mid-ocean ridge hydrothermal circulation inferred from our calculations. The ridge axis is perpendicular to this figure. The circulation is prevented from penetrating into the heat source area due to the formation of the stagnant layer.

salinity contrast decreases as the ratio of the relative velocity to the mean velocity increases in the parameter range of this study. When this ratio is much more than unity, the salinity contrast vanishes because the separation of the two layers becomes so complete that brine cannot be entrained in upwelling fluids. Figure 13 illustrates our view of mid-ocean ridge hydrothermal circulation including the phase separation of seawater. This picture is different from Bischoff and Rosenbauer (1989)'s in that the lower layer is stagnant.

Our model provides a consistent picture of hydrothermal circulation with phase separation. The validity of our model can be tested by measuring salinities of off-axis discharge fluids, which are expected to be counterparts of saline onaxis fluids. Although compositions of off-axis fluids are difficult to measure, they carry valuable information about the total budget of heat and materials.

Acknowledgments. The authors thank J. Cann for helpful discussions and encouragement. The are grateful to S. Schoofs, S. Wallis, and an anonymous reviewer for helpful comments. This work is funded by MEXT (Ministry of Education, Culture, Sports, Science and Technology, Japan) through Special Coordination Fund "Archaean Park; International research project on interaction between sub-vent biosphere and geo-environment" project. This work is also supported by Research Fellowship of the Japan Society for the Promotion of Science for Young Scientists (Y.K.). Part of this work was carried out during S.Y.'s stay at the University of Leeds, which was supported by the British Council Fellowship.

\section{References}

Anderko, K. and K. S. Pitzer, Equation-of-state representation of phase equilibria and volumetric properties of the system $\mathrm{NaCl}-\mathrm{H}_{2} \mathrm{O}$ above 573 K, Geochim. Cosmochim. Acta, 57, 1657-1680, 1993.

Bai, W. M., W. Y. Xu, and R. P. Lowell, The dynamics of submarine geothermal heat pipes, Geophys. Res. Lett., 30, doi:10.1029/2002GL016176, 2003.

Batchelor, G. K., An Introduction to Fluid Dynamics, 635 pp., Cambridge University Press, Cambridge, 1967.

Bear, J., Dynamics of Fluids in Porous Media, 764 pp., Dover, New York, 1988.

Becker, K., Measurements of the permeability of the sheeted dikes in Hole 504B, ODP LEG 111, Proc. Ocean Dril. Prog., Sci. Results, 111, $317-$ $325,1989$.

Berndt, M. E. and W. E. Seyfried, Jr., Boron, bromine, and other trace elements as clues to the fate of chlorine in mid-ocean ridge vent fluids, Geochim. Cosmochim. Acta, 54, 2235-2245, 1990.

Bischoff, J. L. and R. J. Rosenbauer, Salinity variations in submarine hydrothermal systems by layered double-diffusive convection, J. Geol., 97,
613-623, 1989.

Blankenbach, B., F. Busse, U. Christensen, L. Cserepes, D. Gunkel, U. Hansen, H. Harder, G. Jarvis, M. Koch, G. Marquart, D. Moore, P. Olson, H. Schmeling, and T. Schnaubelt, A Benchmark comparison for mantle convection codes, Geophys. J. Int., 98, 23-38, 1989.

Butterfield, D. A., G. J. Massoth, R. E. McDuff, J. E. Lupton, and M. D. Lilley, Geochemistry of hydrothermal fluids from Axial Seamount Hydrothermal Emissions Study vent field, Juan de Fuca ridge: subseafloor boiling and subsequent fluid-rock interaction, J. Geophys. Res., 95, 12895-12921, 1990.

Cherkaoui, A. S. M. and W. S. D. Wilcock, Characteristics of high Rayleigh number two-dimensional convection in an open-top porous layer heated from below, J. Fluid Mech., 394, 241-260, 1999.

Christensen, U., Convection with pressure- and temperature-dependent nonNewtonian rheology, Geophys. J. R. astr. Soc., 77, 343-384, 1984.

Elder, J. W., Steady free convection in a porous medium heated from below, J. Fluid Mech., 27, 29-48, 1967.

Fehn, U., K. E. Green, R. P. Von Herzen, and L. M. Cathles, Numerical models for the hydrothermal field at the Galapagos spreading center, $J$. Geophys. Res., 88, 1033-1048, 1983.

Fisher, A. T., Permeability within basaltic oceanic crust, Rev. Geophys., 36, 143-182, 1998.

Fontaine, F. Jh., M. Rabinowicz, and J. Boulègue, Permeability changes due to mineral diagenesis in fractured crust: Implications for hydrothermal circulation at mid-ocean ridges, Earth Planet. Sci. Lett., 184, 407-425, 2001.

Fornari, D. J. and R. W. Embley, Tectonic and volcanic controls on hydrothermal processes at the mid-ocean ridge: An overview based on near-bottom and submersible studies, in Seafloor Hydrothermal Systems: Physical, Chemical, Biological, and Geological Interactions, Geophys. Monogr., vol. 91, edited by S. E. Humphris et al., pp. 1-46, AGU, Washington, D.C., 1995.

Fournier, R. O., Conceptual models of brine evolution in magmatichydrothermal systems, U. S. Geol. Surv. Prof. Pap., 1350, 1487-1506, 1987.

Haar, J. L., J. S. Gallagher, and G. S. Kell, NBS/NRC Steam Tables: Thermodynamic and Transport Properties and Computer Programs for Vapor and Liquid States of Water in SI units, 320 pp., Hemisphere, New York, 1984.

Ishii, M., Thermo-Fluid Dynamic Theory of Two-Phase Flow, 248 pp., Eyrolles, Paris, 1975.

Jupp, T. and A. Schultz, A thermodynamic explanation for black smoker temperatures, Nature, 403, 880-883, 2000.

Kelley, D. S., P. T. Robinson, and J. G. Malpas, Processes of brine generation and circulation in the oceanic crust: Fluid inclusion evidence from the Troodos Ophiolite, Cyprus, J. Geophys. Res., 97, 9307-9322, 1992.

Kelley, D. S., J. A. Baross, and J. R. Delaney, Volcanoes, fluids, and life at mid-ocean ridge spreading centers, Annu. Rev. Earth Planet. Sci., 30, 385-491, 2002.

Koshizuka, S., Computational Fluid Dynamics, 223 pp., Baifukan, Tokyo, 1997 (in Japanese).

Lasaga, A. C., Kinetics Theory in the Earth Sciences, 811 pp, Princeton University Press, Princeton, New Jersey, 1998.

Lowell, R. P., Modeling continental and submarine hydrothermal systems, Rev. Geophys., 29, 457-476, 1991.

Lowell, R. P. and L. N. Germanovich, Evolution of a brine-saturated layer at the base of a ridge-crest hydrothermal system, J. Geophys. Res., 102, 10245-10255, 1997

Lowell, R. P. and W. Xu, Sub-critical two-phase seawater convection near a dike, Earth Planet. Sci. Lett., 174, 385-396, 2000.

Lowell, R. P., P. A. Rona, and R. P. Von Herzen, Seafloor hydrothermal systems, J. Geophys. Res., 100, 327-352, 1995.

Martin, J. T. and R. P. Lowell, Precipitation of quartz during hightemperature, fracture-controlled hydrothermal upflow at ocean ridges: Equilibrium versus linear kinetics, J. Geophys. Res., 105, 869-882, 2000.

Mével, C. and M. Cannat, Lithospheric stretching and hydrothermal processes in oceanic gabbros from slow-spreading ridges, in Ophiolite Genesis and Evolution of the Oceanic Lithosphere, edited by Tj. Peters et al., pp. 293-312, Kluwer Academic, Dordrecht, 1991.

Nehlig, P., Fracture and permeability analysis in magma-hydrothermal transition zones in the Samail ophiolite (Oman), J. Geophys. Res., 99, 589601, 1994.

Nehlig, P. and T. Juteau, Flow porosities, permeabilities, and preliminary data on fluid inclusions and fossil thermal-gradients in the crustal sequence of the Sumail ophiolite (Oman), Tectonophys., 151, 199-221, 
1988.

Nield, D. A. and A. Bejan, Convection in Porous Media, second edition, 546 pp., Springer-Verlag, New York, 1999.

Palliser, C. and R. McKibbin, A model for deep geothermal brines, I: T $p-X$ state-space description, Transport in Porous Media, 33, 65-80, 1998a.

Palliser, C. and R. McKibbin, A model for deep geothermal brines, III: Thermodynamic properties-Enthalpy and viscosity, Transport in Porous Media, 33, 155-171, 1998 b.

Pitzer, K. S., J. C. Peiper, and R. H. Busey, Thermodynamic properties of aqueous sodium chloride solutions, J. Phys. Chem. Ref. Data, 13, 1-102, 1984.

Raithby, G. D., Skew upstream differencing schemes for problems involving fluid flow, Comp. Methods Appl. Mech. Eng., 9, 153-164, 1976.

Schoofs, S. and U. Hansen, Depletion of a brine layer at the base of ridgecrest hydrothermal systems, Earth Planet. Sci. Lett., 180, 341-353, 2000.

Schoofs, S. and F. J. Spera, Transition to chaos and flow dynamics of thermochemical porous medium convection, Transport in Porous Media, 50, 179-195, 2003.

Schoofs, S., F. J. Spera, and U. Hansen, Chaotic thermohaline convection in low-porosity hydrothermal systems, Earth Planet. Sci. Lett., 174, $213-$ 229, 1999.

Schoofs, S., R. A. Trompert, and U. Hansen, The formation and evolution of layered structures in porous media: Esffects of porosity and mechanical dispersion, Phys. Earth Planet. Int., 118, 205-225, 2000.

Sinton, J. M. and R. S. Detrick, Mid-ocean ridge magma chambers, $J$. Geophys. Res., 97, 197-216, 1992.

Travis, B. J., D. R. Janecky, and N. D. Rosenberg, Three-dimensional simulation of hydrothermal circulation at mid-ocean ridges, Geophys. Res. Lett., 18, 1441-1444, 1991.

Von Damm, K. L., Controls on the chemistry and temporal variability of seafloor hydrothermal fluids, in Seafloor Hydrothermal Systems: Physical, Chemical, Biological, and Geological Interactions, Geophys. Monogr., vol. 91, edited by S. E. Humphris et al., pp. 222-247, AGU, Washington, D.C., 1995.

Von Damm, K. L., M. D. Lilley, W. C. Shanks, III, M. Brockington, A. M. Brey, K. M. O'Grady, E. Olson, A. Graham, G. Proskurowski, and the SouEPR Science Party, Extraordinary phase separation and segregation in vent fluids from the southern East Pacific Rise, Earth Planet. Sci. Lett., 206, 365-378, 2003.

Wilcock, W. S. D., Cellular convection models of mid-ocean ridge hydrothermal circulation and the temperatures of black smoker fluids, $J$. Geophys. Res., 103, 2585-2596, 1998.

Y. Kawada (e-mail: kawada@eps.nagoya-u.ac.jp), S. Yoshida (e-mail: yoshida@eps.nagoya-u.ac.jp), S. Watanabe (e-mail: seicoro@eps.nagoyau.ac.jp) 